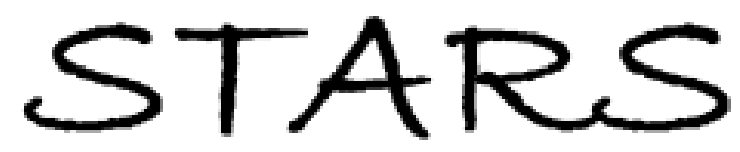

University of Central Florida

STARS

$1-1-2009$

\title{
Optimal Stopping Problem for Stochastic Differential Equations With Random Coefficients
}

Mou-Hsiung Chang

Tao Pang

Jiongmin Yong

University of Central Florida

Find similar works at: https://stars.library.ucf.edu/facultybib2000

University of Central Florida Libraries http://library.ucf.edu

This Article is brought to you for free and open access by the Faculty Bibliography at STARS. It has been accepted for inclusion in Faculty Bibliography 2000 s by an authorized administrator of STARS. For more information, please contact STARS@ucf.edu.

\section{Recommended Citation}

Chang, Mou-Hsiung; Pang, Tao; and Yong, Jiongmin, "Optimal Stopping Problem for Stochastic Differential Equations With Random Coefficients" (2009). Faculty Bibliography 2000s. 1400.

https://stars.library.ucf.edu/facultybib2000/1400

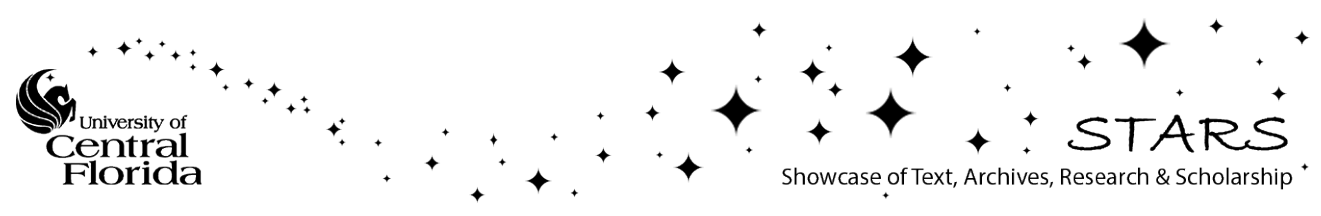




\title{
OPTIMAL STOPPING PROBLEM FOR STOCHASTIC DIFFERENTIAL EQUATIONS WITH RANDOM COEFFICIENTS*
}

\author{
MOU-HSIUNG CHANG ${ }^{\dagger}$, TAO PANG ${ }^{\ddagger}$, AND JIONGMIN YONG $\$$
}

\begin{abstract}
An optimal stopping problem for stochastic differential equations with random coefficients is considered. The dynamic programming principle leads to a Hamiltion-Jacobi-Bellman equation, which, for the current case, is a backward stochastic partial differential variational inequality (BSPDVI, for short) for the value function. Well-posedness of such a BSPDVI is established, and a verification theorem is proved.
\end{abstract}

Key words. optimal stopping, random coefficients, dynamic programming principle, backward stochastic partial differential variational inequality, verification theorem

AMS subject classifications. 49L20, 93E20

DOI. $10.1137 / 070705726$

1. Introduction. Throughout this paper, we let $(\Omega, \mathcal{F}, \mathbb{F}, \mathbb{P})$ be a complete filtered probability space on which a $d$-dimensional standard Brownian motion $W(\cdot)$ is defined, with $\mathbb{F} \equiv\left\{\mathcal{F}_{t}\right\}_{t \geq 0}$ being its natural filtration augmented by all the $\mathbb{P}$-null sets in $\mathcal{F}$. Let $\mathcal{S}[0, T]$ be the set of all $\mathbb{F}$-stopping times taking values in $[0, T]$. For any $\tau_{1}, \tau_{2} \in \mathcal{S}[0, T]$, with $\tau_{1} \leq \tau_{2}$ almost surely and $\mathbb{P}\left\{\tau_{1}<\tau_{2}\right\}>0$, let

$$
\left\{\begin{array}{l}
\mathcal{S}\left[\tau_{1}, \tau_{2}\right] \triangleq\left\{\tau \in \mathcal{S}[0, T] \mid \tau_{1} \leq \tau \leq \tau_{2} \text { a.s. }\right\} \\
\mathcal{S}\left(\tau_{1}, \tau_{2}\right] \triangleq\left\{\tau \in \mathcal{S}\left[\tau_{1}, \tau_{2}\right] \mid \tau_{1}<\tau \text { a.s. } \quad \text { on }\left\{\tau_{1}<\tau_{2}\right\}\right\}, \\
\mathcal{S}\left[\tau_{1}, \tau_{2}\right) \triangleq\left\{\tau \in \mathcal{S}\left[\tau_{1}, \tau_{2}\right] \mid \tau<\tau_{2} \text { a.s. } \text { on }\left\{\tau_{1}<\tau_{2}\right\}\right\} \\
\mathcal{S}\left(\tau_{1}, \tau_{2}\right) \triangleq\left\{\tau \in \mathcal{S}\left[\tau_{1}, \tau_{2}\right] \mid \tau_{1}<\tau<\tau_{2} \text { a.s. } \text { on }\left\{\tau_{1}<\tau_{2}\right\}\right\} .
\end{array}\right.
$$

Next, for any $s \in \mathcal{S}[0, T]$ and $p \geq 1$, denote

$$
\mathcal{X}_{s}^{p} \equiv L_{\mathcal{F}_{s}}^{p}\left(\Omega ; \mathbb{R}^{n}\right) \triangleq\left\{\xi: \Omega \rightarrow \mathbb{R}^{n} \mid \xi \text { is } \mathcal{F}_{s^{-}} \text {measurable, } \mathbb{E}|\xi|^{p}<\infty\right\}
$$

For any $s \in \mathcal{S}[0, T)$ and $\xi \in \mathcal{X}_{s}^{p}$, consider the following stochastic differential equation (SDE, for short):

$$
\left\{\begin{array}{l}
d X(t)=b(t, X(t)) d t+\sigma(t, X(t)) d W(t), \quad t \in[s, T] \\
X(s)=\xi
\end{array}\right.
$$

where $b:[0, T] \times \mathbb{R}^{n} \times \Omega \rightarrow \mathbb{R}^{n}$ and $\sigma:[0, T] \times \mathbb{R}^{n} \times \Omega \rightarrow \mathbb{R}^{n \times d}$ are given maps. We refer to the above as the state equation. Under proper conditions (which will be

* Received by the editors October 18, 2007; accepted for publication (in revised form) November 10, 2008; published electronically February 25, 2009. This work is supported in part by grant W911NF04-D-0003 from the U.S. Army Research Office and by NSF grant DMS-0604309.

http://www.siam.org/journals/sicon/48-2/70572.html

${ }^{\dagger}$ Mathematics Division, U.S. Army Research Office, Research Triangle Park, NC 27709 (mouhsiung.chang@us.army.mil).

${ }^{\ddagger}$ Department of Mathematics, Center for Research in Scientific Computation, North Carolina State University, Raleigh, NC 27695 (tpang@unity.ncsu.edu).

$\S$ Department of Mathematics, University of Central Florida, Orlando, FL 32816 (jyong@mail.ucf. edu). 
assumed shortly), the above SDE admits a unique strong solution $X(\cdot) \equiv X(\cdot ; s, \xi)$. Introduce the following cost functional:

$$
J_{s, \xi}(\tau)=\mathbb{E}\left[\int_{s}^{\tau} g(t, X(t ; s, \xi)) d t+h(\tau, X(\tau ; s, \xi)) \mid \mathcal{F}_{s}\right], \quad \tau \in \mathcal{S}[s, T],
$$

where $g, h:[0, T] \times \mathbb{R}^{n} \times \Omega \rightarrow[0, \infty)$ are some given nonnegative maps satisfying proper conditions. The two terms on the right-hand side of (1.4) represent the running cost and the terminal cost, respectively. We point out that all the involved maps $b, \sigma, g$, and $h$ in our discussion are allowed to be random. With the above setting, we can now pose the following optimal stopping problem.

Problem (S). For given $s \in \mathcal{S}[0, T)$ and $\xi \in \mathcal{X}_{s}^{p}$, find the smallest $\bar{\tau} \in \mathcal{S}[s, T]$ such that

$$
J_{s, \xi}(\bar{\tau})=\inf _{\tau \in \mathcal{S}[s, T]} J_{s, \xi}(\tau) \equiv V(s, \xi) .
$$

Any $\bar{\tau} \in \mathcal{S}[s, T]$ satisfying (1.5) is referred to as an optimal stopping time, and the smallest one is referred to as the smallest optimal stopping time. We compatibly define

$$
V(T, \xi)=h(T, \xi) \quad \forall \xi \in \mathcal{X}_{s}^{p} .
$$

Random field $V(\cdot, \cdot)$ defined by (1.5)-(1.6) is called the value function of Problem (S). We point out that for the maps $g$ and $h$, a nonnegativity condition can be relaxed to the boundedness from below. On the other hand, it is not hard to see that if $h=0$ and $g>0$, then any optimal stopping time of Problem (S) must be the smallest one. But, in general, the optimal stopping time of Problem (S) is not necessarily unique (one can modify Example D.14 of [14]). Hence, to be definite, our Problem (S) is to find the smallest optimal stopping time. We also note that, due to the fact that the coefficients are allowed to be random and our cost functional is defined by a conditional expectation, our value function $V(\cdot, \cdot)$ is actually a random field.

In the case where all the coefficients are deterministic, one can prove the dynamic programming principle which leads to a partial differential variational inequality (PDVI, for short), as the corresponding HJB equation for the value function (which is deterministic). Moreover, it can be shown that the value function is the unique viscosity solution to the PDVI. In the case where the diffusion is uniformly nondegenerate, the value function is the (unique) classical solution of the PDVI, provided that some mild smoothness conditions are assumed for the coefficients. On the other hand, one can independently establish the well-posedness of the corresponding PDVI, as well as a verification theorem. These will then provide a solution to the original optimal stopping time problem (see [2] and the references cited therein).

We also note that, by some pure probabilistic approach, one can study an optimal stopping time problem for general continuous-time stochastic processes. Optimal stopping time is characterized by means of the so-called Snell's envelope, super martingale, and so on, without using the dynamic programming principle. In such an approach, no HJB equation is involved, which is natural because no dynamic equation is assumed for the considered stochastic processes (see [14]). We refer to [9], [10], [25], [3], [24], [8], [28], [22], [29], [18], [1], [5], [23], [4], [27], [7], [11] for relevant results on stochastic optimal stopping and optimal control problems.

For the problem under our consideration, since we have more structures on the stochastic process (satisfying an SDE, etc.), it is expected to have more detailed characterization on the optimal stopping time. On the other hand, due to the randomness 
of the coefficients, the usual technique of the dynamic programming principle together with theories of PDVIs, do not directly apply. In this paper, inspired by [21], we will formally derive the corresponding HJB equation for the value function $V(\cdot, \cdot)$, which is now a backward stochastic partial differential variational inequality (BSPDVI, for short). Using a result of semilinear backward stochastic partial differential equations (BSPDEs, for short) from [26], together with a standard penalty technique for (deterministic) PDVIs (see [12]), we will obtain the well-posedness of our BSPDVI in a certain sense. At the same time, a verification theorem will be established, which says that, under proper conditions, the solution to the BSPDVI coincides with the value function of Problem (S). Then an optimal stopping time can be characterized. See [20] for some results concerning backward stochastic variational inequalities in an abstract framework.

The rest of the paper is organized as follows: Some preliminary results, including certain basic properties of the value function will be presented in section 2 . In section 3, we will formally derive the BSPDVI and formally prove a verification theorem. Notions of adapted solutions will be introduced in section 4. The well-posedness of the BSPDVI will be established in section 5. Finally, in section 6, the adapted weak solution of the BSPDVI will be identified as the value function of Problem (S).

2. Some preliminary results. In this section, we are going to present some preliminary results related to value function $V(\cdot, \cdot)$ of Problem (S). To begin with, for any $p \geq 1, s \in \mathcal{S}[0, T)$, and $\tau \in \mathcal{S}(s, T]$, we let $L_{\mathbb{F}}^{p}\left(\Omega ; C\left([s, \tau] ; \mathbb{R}^{n}\right)\right)$ be the set of all processes $\varphi:[s, \tau] \rightarrow \mathbb{R}^{n}$ having continuous paths and

$$
\mathbb{E}\left[\sup _{t \in[s, \tau]}|\varphi(t)|^{p}\right]<\infty .
$$

It is clear that $L_{\mathbb{F}}^{p}\left(\Omega ; C\left([s, \tau] ; \mathbb{R}^{n}\right)\right)$ is a Banach space. Next, for $p \geq 1$, we denote (recall (1.2))

$$
\mathcal{D}^{p}=\left\{(s, \xi) \in \mathcal{S}[0, T] \times \mathcal{X}_{T}^{p} \mid s \in \mathcal{S}[0, T], \xi \in \mathcal{X}_{s}^{p}\right\} .
$$

Now, we introduce the following standing assumption concerning the coefficients of state equation (1.3).

Assumption (H1). Maps $b:[0, T] \times \mathbb{R}^{n} \times \Omega \rightarrow \mathbb{R}^{n}$ and $\sigma:[0, T] \times \mathbb{R}^{n} \times \Omega \rightarrow \mathbb{R}^{n \times d}$ are measurable and they satisfy the following:

(a) For each $x \in \mathbb{R}^{n}, t \mapsto(b(t, x), \sigma(t, x))$ is $\mathbb{F}$-progressively measurable and for some $p>1$,

$$
\mathbb{E}\left(\int_{0}^{T}|b(t, 0)| d t\right)^{p}+\mathbb{E}\left(\int_{0}^{T}|\sigma(t, 0)|^{2} d t\right)^{\frac{p}{2}}<\infty .
$$

(b) There exists an $L>0$ such that

$$
\begin{array}{r}
|b(t, x, \omega)-b(t, y, \omega)|+|\sigma(t, x, \omega)-\sigma(t, y, \omega)| \leq L|x-y|, \\
\text { a.e. } t \in[0, T], \forall x, y \in \mathbb{R}^{n}, \text { a.s. } \omega \in \Omega .
\end{array}
$$

Concerning the maps appearing in the cost functional, we introduce the following assumption.

Assumption (H2). Maps $g, h:[0, T] \times \mathbb{R}^{n} \times \Omega \rightarrow[0, \infty)$ are measurable, and they satisfy the following: 
(a) For each $x \in \mathbb{R}^{n}, t \mapsto(g(t, x), h(t, x))$ is $\mathbb{F}$-progressively measurable; for each $x \in \mathbb{R}^{n}$ and a.s. $\omega \in \Omega, t \mapsto h(t, x)$ is continuous, and

$$
\mathbb{E}\left[\int_{0}^{T} g(t, 0) d t+\sup _{t \in[0, T]} h(t, 0)\right]<\infty
$$

(b) There exists an $L>0$ such that

$$
\begin{array}{r}
|g(t, x, \omega)-g(t, y, \omega)|+|h(t, x, \omega)-h(t, y, \omega)| \leq L|x-y|, \\
\text { a.e. } t \in[0, T], \forall x, y \in \mathbb{R}^{n}, \text { a.s. } \omega \in \Omega,
\end{array}
$$

and there exists a continuous nondecreasing function $\rho:[0, \infty) \rightarrow[0, \infty)$, with $\rho(0)=$ 0 such that

$$
|h(t, x, \omega)-h(s, x, \omega)| \leq(1+|x|) \rho(|t-s|), \quad \forall t, s \in[0, T], x \in \mathbb{R}^{n}, \quad \text { a.s. }
$$

The following result is pretty standard (see [13]).

Proposition 2.1. Let (H1) hold. Then, for each $(s, \xi) \in \mathcal{D}^{p}$, state equation (1.3) admits a unique (strong) solution $X(\cdot) \equiv X(\cdot ; s, \xi) \in L_{\mathbb{F}}^{p}\left(\Omega ; C\left([s, T] ; \mathbb{R}^{n}\right)\right)$. Moreover,

$$
\begin{gathered}
\mathbb{E}\left[\sup _{t \in[s, T]}|X(t ; s, \xi)|^{p} \mid \mathcal{F}_{s}\right] \leq C\left(1+|\xi|^{p}\right) \quad \forall(s, \xi) \in \mathcal{D}^{p}, \\
\mathbb{E}\left[\sup _{t \in[s, T]}|X(t ; s, \xi)-X(t ; s, \bar{\xi})|^{p} \mid \mathcal{F}_{s}\right] \leq C|\xi-\bar{\xi}|^{p} \quad \forall(s, \xi),(s, \bar{\xi}) \in \mathcal{D}^{p},
\end{gathered}
$$

and when $p>1$,

$$
\begin{gathered}
(2.8) \quad \mathbb{E}\left[\sup _{t \in[s, \tau]}|X(t ; s, \xi)-\xi|^{\bar{p}} \mid \mathcal{F}_{s}\right] \leq C\left(1+|\xi|^{\bar{p}}\right)\left\{\mathbb { E } \left[|\tau-s|^{\left.\left.\frac{p \bar{p}}{2(p-\bar{p})} \mid \mathcal{F}_{s}\right]\right\}^{\frac{p-\bar{p}}{p}},}\right.\right. \\
\forall(s, \xi) \in \mathcal{D}^{p}, \tau \in \mathcal{S}[s, T], \quad \bar{p} \in[1, p), \\
\mathbb{E}\left[\sup _{t \in[s \vee \bar{s}, T]}|X(t ; s, \xi)-X(t ; \bar{s}, \xi)|^{\bar{p}} \mid \mathcal{F}_{s \wedge \bar{s}}\right] \leq C\left(1+|\xi|^{\bar{p}}\right)\left\{\mathbb { E } \left[|s-\bar{s}|^{\left.\left.\frac{p \bar{p}}{2(p-\bar{p})} \mid \mathcal{F}_{s \wedge \bar{s}}\right]\right\}^{\frac{p-\bar{p}}{p}},}\right.\right. \\
\forall s, \bar{s} \in \mathcal{S}[0, T], \quad \xi \in \mathcal{X}_{s \wedge \bar{s}}^{p}, \quad \bar{p} \in[1, p),
\end{gathered}
$$

hereafter, $C>0$ represents a generic constant which can be different from line to line.

A simple consequence of the above is that

$$
(t, X(t ; s, \xi)) \in \mathcal{D}^{p}, \quad t \in \mathcal{S}[s, T], \quad \forall(s, \xi) \in \mathcal{D}^{p} .
$$

We also note that if both $s, \bar{s} \in[0, T]$ are deterministic, then

$$
\mathbb{E}\left[\sup _{t \in[s \vee \bar{s}, T]}|X(t ; s, \xi)-X(t ; \bar{s}, \xi)|^{p} \mid \mathcal{F}_{s \wedge \bar{s}}\right] \leq C\left(1+|\xi|^{p}\right)|s-\bar{s}|^{\frac{p}{2}} \quad \forall \xi \in \mathcal{X}_{s \wedge \bar{s}}^{p}
$$

The following proposition collects some basic results concerning value function $V(\cdot, \cdot)$.

Proposition 2.2. Let (H1)-(H2) hold. Then

Copyright (C) by SIAM. Unauthorized reproduction of this article is prohibited. 
(i) For any $(s, \xi) \in \mathcal{D}^{p}$ and $\tau \in \mathcal{S}[s, T], J_{s, \xi}(\tau)$ is a well-defined $\mathcal{F}_{s}$-measurable random variable. Moreover, there exists a $\bar{\tau}(s, \xi) \in \mathcal{S}[s, T]$ such that

$$
V(s, \xi) \equiv \inf _{\tau \in \mathcal{S}[s, T]} J_{s, \xi}(\tau)=J_{s, \xi}(\bar{\tau}(s, \xi))
$$

Consequently, for any $(s, \xi) \in \mathcal{D}^{p}, V(s, \xi)$ is $\mathcal{F}_{s}$-measurable.

(ii) Value function $V(\cdot, \cdot)$ satisfies the following:

$$
\begin{gathered}
|V(s, \xi)| \leq C(1+|\xi|) \quad \forall(s, \xi) \in \mathcal{D}^{p}, \\
|V(s, \xi)-V(s, \bar{\xi})| \leq C|\xi-\bar{\xi}| \quad \forall(s, \xi),(s, \bar{\xi}) \in \mathcal{D}^{p},
\end{gathered}
$$

and when $p>1$,

$$
\begin{aligned}
\mid \mathbb{E}[V(s, \xi) & \left.-V(\bar{s}, \xi) \mid \mathcal{F}_{s \wedge \bar{s}}\right] \mid \leq C(1+|\xi|)\left\{\left[\mathbb{E}\left(|s-\bar{s}|^{\frac{p}{2(p-1)}} \mid \mathcal{F}_{s \wedge \bar{s}}\right)\right]^{\frac{p-1}{p}}\right. \\
+ & \left.\mathbb{E}\left[\rho(|s-\bar{s}|)+|s-\bar{s}| \mid \mathcal{F}_{s \wedge \bar{s}}\right]\right\}, \quad \forall s, \bar{s} \in \mathcal{S}[0, T], \xi \in \mathcal{X}_{s \wedge \bar{s}}^{p}
\end{aligned}
$$

(iii) For any $s \in \mathcal{S}[0, T)$ and any $\varphi(\cdot) \in L_{\mathbb{F}}^{1}\left(\Omega ; C\left([0, T] ; \mathbb{R}^{n}\right)\right.$, map $t \mapsto V(t, \varphi(t))$ is $\mathbb{F}$-adapted on $[s, T]$. In particular, for any $(s, \xi) \in \mathcal{D}^{p}$, map $t \mapsto V(t, X(t ; s, \xi))$ is $\mathbb{F}$-adapted.

Proof. (i) By Proposition 2.1 and (H2), we see that, for any fixed $(s, \xi) \in \mathcal{D}^{p}$,

$$
\left|J_{s, \xi}(\tau)\right| \leq \mathbb{E}\left[\int_{s}^{\tau}|g(r, X(r ; s, \xi))| d r+|h(\tau, X(\tau ; s, \xi))| \mid \mathcal{F}_{s}\right] \leq C(1+|\xi|), \quad \tau \in \mathcal{S}[s, T] .
$$

Hence, $J_{s, \xi}(\tau)$ is a well-defined $\mathcal{F}_{s}$-measurable random variable. Next, it is clear that $t \mapsto J_{s, \xi}(t)$ is continuous. Thus, by Theorem D.12 of [14] (see also [8]), with a minor modification, we have the existence of an optimal stopping time $\bar{\tau}(s, \xi)$ for Problem (S).

(ii) For any $(s, \xi),(s, \bar{\xi}) \in \mathcal{D}^{p}$, by (H2) and Proposition 2.1, we can get

$$
\begin{array}{r}
\left|J_{s, \xi}(\theta)-J_{s, \bar{\xi}}(\theta)\right| \leq \mathbb{E}\left[\int_{s}^{\theta}|g(r, X(r ; s, \xi))-g(r, X(r ; s, \bar{\xi}))| d r\right. \\
\left.+|h(\theta, X(\theta ; s, \xi))-h(\theta, X(\theta ; s, \bar{\xi}))| \mid \mathcal{F}_{s}\right] \\
\leq C \mathbb{E}\left[\sup _{t \in[s, \theta]}|X(t ; s, \xi)-X(t ; s, \bar{\xi})| \mid \mathcal{F}_{s}\right] \leq C|\xi-\bar{\xi}| \quad \forall \theta \in \mathcal{S}[s, T],
\end{array}
$$

with $C>0$ being an absolute constant. Hence, (2.14) follows. Next, let $s, \bar{s} \in \mathcal{S}[0, T]$,

Copyright $@$ by SIAM. Unauthorized reproduction of this article is prohibited. 
$\xi \in \mathcal{X}_{s \wedge \bar{s}}^{p}$, and $\theta \in \mathcal{S}[s \wedge \bar{s}, T]$. Observe the following:

$$
\begin{aligned}
& \left|\mathbb{E}\left[J_{s, \xi}(s \vee \theta)-J_{\bar{s}, \xi}(\bar{s} \vee \theta) \mid \mathcal{F}_{s \wedge \bar{s}}\right]\right| \\
& =\mid \mathbb{E}[h(s \vee \theta, X(s \vee \theta ; s, \xi))-h(\bar{s} \vee \theta, X(\bar{s} \vee \theta ; \bar{s}, \xi)) \\
& \left.+\int_{s}^{s \vee \theta} g(t, X(t ; s, \xi)) d t-\int_{\bar{s}}^{\bar{s} \vee \theta} g(t, X(t ; \bar{s}, \xi)) d t \mid \mathcal{F}_{s \wedge \bar{s}}\right] \mid \\
& =\mid \mathbb{E}\left\{I_{(s \vee \bar{s} \leq \theta)}[h(\theta, X(\theta ; s, \xi))-h(\theta, X(\theta ; \bar{s}, \xi))]\right. \\
& +I_{(s<\theta<\bar{s})}[h(\theta, X(\theta ; s, \xi))-h(\bar{s}, \xi)]+I_{(\bar{s}<\theta<s)}[h(s, \xi)-h(\theta, X(\theta ; \bar{s}, \xi))] \\
& +I_{(s \vee \bar{s} \leq \theta)}\left[\int_{s}^{s \vee \bar{s}} g(t, X(t ; s, \xi)) d t-\int_{\bar{s}}^{s \vee \bar{s}} g(t, X(t ; \bar{s}, \xi)) d t\right. \\
& \left.+\int_{s \vee \bar{s}}^{\theta}(g(t, X(t ; s, \xi)) d t-g(t, X(t ; \bar{s}, \xi))) d t\right] \\
& \left.+I_{(s<\theta<\bar{s})} \int_{s}^{\theta} g(t, X(t ; s, \xi)) d t-I_{(\bar{s}<\theta<s)} \int_{\bar{s}}^{\theta} g(t, X(t ; \bar{s}, \xi)) d t \mid \mathcal{F}_{s \wedge \bar{s}}\right\} \mid \\
& \leq \mathbb{E}\left\{I_{(s \vee \bar{s} \leq \theta)} L|X(\theta ; s, \xi)-X(\theta ; \bar{s}, \xi)|+I_{(s<\theta<\bar{s})}[L|X(\theta ; s, \xi)-\xi|+(1+|\xi|) \rho(|s-\bar{s}|)]\right. \\
& +I_{(\bar{s}<\theta<s)}[L|X(\theta ; \bar{s}, \xi)-\xi|+(1+|\xi|) \rho(|s-\bar{s}|)] \\
& +C\left(1+\sup _{t \in[s, T]}|X(t ; s, \xi)|+\sup _{t \in[\bar{s}, T]}|X(t ; \bar{s}, \xi)|\right)|s-\bar{s}| \\
& \left.+I_{(s \vee \bar{s} \leq \theta)} \int_{s \vee \bar{s}}^{\theta} L|X(t ; s, \xi)-X(t ; \bar{s}, \xi)| d t \mid \mathcal{F}_{s \wedge \bar{s}}\right\} \\
& \leq C\left\{(1+|\xi|)\left[\mathbb{E}\left(|s-\bar{s}|^{\frac{p}{2(p-1)}} \mid \mathcal{F}_{s \wedge \bar{s}}\right)\right]^{\frac{p-1}{p}}+(1+|\xi|) \mathbb{E}\left[\rho(|s-\bar{s}|) \mid \mathcal{F}_{s \wedge \bar{s}}\right]\right. \\
& \left.+(1+|\xi|) \mathbb{E}\left[|s-\bar{s}| \mid \mathcal{F}_{s \wedge \bar{s}}\right]+(1+|\xi|)\left[\mathbb{E}\left(|s-\bar{s}|^{\frac{p}{2(p-1)}} \mid \mathcal{F}_{s \wedge \bar{s}}\right)\right]^{\frac{p-1}{p}}\right\} \\
& \leq C(1+|\xi|)\left\{\left[\mathbb{E}\left(|s-\bar{s}|^{\frac{p}{2(p-1)}} \mid \mathcal{F}_{s \wedge \bar{s}}\right)\right]^{\frac{p-1}{p}}+\mathbb{E}\left[\rho(|s-\bar{s}|)+|s-\bar{s}| \mid \mathcal{F}_{s \wedge \bar{s}}\right]\right\} .
\end{aligned}
$$

Hence, taking $\theta=\bar{\tau}(\bar{s}, \xi)$, we obtain (note $\bar{\tau}(\bar{s}, \xi) \geq \bar{s}$ )

$$
\begin{aligned}
& \mathbb{E}\left[V(s, \xi)-V(\bar{s}, \xi) \mid \mathcal{F}_{s \wedge \bar{s}}\right] \leq \mathbb{E}\left[J_{s, \xi}(s \vee \bar{\tau}(\bar{s}, \xi))-J_{\bar{s}, \xi}(\bar{\tau}(\bar{s}, \xi)) \mid \mathcal{F}_{s \wedge \bar{s}}\right] \\
& \leq C(1+|\xi|)\left\{\left[\mathbb{E}\left(|s-\bar{s}|^{\frac{p}{2(p-1)}} \mid \mathcal{F}_{s \wedge \bar{s}}\right)\right]^{\frac{p-1}{p}}+\mathbb{E}\left[\rho(|s-\bar{s}|)+|s-\bar{s}| \mid \mathcal{F}_{s \wedge \bar{s}}\right]\right\} .
\end{aligned}
$$

Exchanging the roles of $s$ and $\bar{s}$, we obtain (2.15).

(iii) is clear.

3. Principle of optimality and BSPDVI. We now would like to formally derive the equation that value function $V(\cdot, \cdot)$ should satisfy. To this end, we first state the following principle of optimality.

TheOrem 3.1. Let (H1)-(H2) hold.

Copyright $@$ by SIAM. Unauthorized reproduction of this article is prohibited. 
(i) For any $(s, \xi) \in \mathcal{D}^{p}$,

$$
V(s, \xi) \leq h(s, \xi) \quad \text { a.s. }
$$

and

$$
V(s, \xi) \leq \inf _{\tau \in \mathcal{T}[s, T]} \mathbb{E}\left[\int_{s}^{\tau} g(r, X(r ; s, \xi)) d r+V(\tau, X(\tau ; s, \xi)) \mid \mathcal{F}_{s}\right] \quad \text { a.s. }
$$

(ii) For any $(s, \xi) \in \mathcal{D}^{p}$, if $\bar{\theta} \in \mathcal{S}[s, T]$ is an optimal stopping time of Problem (S) for the initial point $(s, \xi)$, then

$$
V(\bar{\theta}, X(\bar{\theta} ; s, \xi))=h(\bar{\theta}, X(\bar{\theta} ; s, \xi)) \quad \text { a.s. }
$$

Hence, the following is the smallest optimal stopping time of Problem (S) corresponding to $(s, \xi)$ :

$$
\bar{\tau}(s, \xi)=\inf \{t \in[s, T] \mid V(t, X(t ; s, \xi))=h(t, X(t ; s, \xi))\} .
$$

Moreover,

$$
\mathbb{P}(\{\bar{\tau}(s, \xi)>s\} \Delta\{V(s, \xi)<h(s, \xi)\})=0,
$$

where $A \Delta B=(A \backslash B) \cup(B \backslash A)$, for any $A, B \in \mathcal{F}$, and

$$
\begin{gathered}
V(\theta, X(\theta ; s, \xi))=\mathbb{E}\left[\int_{\theta}^{\tau} g(r, X(r ; s, \xi)) d r+V(\tau, X(\tau ; s, \xi)) \mid \mathcal{F}_{\theta}\right], \\
\forall \theta \in \mathcal{S}[s, \bar{\tau}(s, \xi)], \quad \tau \in \mathcal{S}[\theta, \bar{\tau}(s, \xi)], \quad \text { a.s. }
\end{gathered}
$$

The above results are basically known (see [8]). For the readers's convenience, we sketch a proof in the appendix.

Note that (3.5) tells us the following: Up to a $\mathbb{P}$-null set, one has

$$
\{\bar{\tau}(s, \xi)>s\}=\{V(s, \xi)<h(s, \xi)\} .
$$

Consequently, up to a $\mathbb{P}$-null set, the following holds:

$$
\{\bar{\tau}(s, \xi)=s\}=\{V(s, \xi)=h(s, \xi)\} .
$$

On the other hand, (3.2) implies that

$$
\begin{aligned}
& V(\theta, X(\theta ; s, \xi))+\int_{s}^{\theta} g(r, X(r ; s, \xi)) d r \\
& \leq \mathbb{E}\left[V(\tau, X(\tau ; s, \xi))+\int_{s}^{\tau} g(r, X(r ; s, \xi)) d r \mid \mathcal{F}_{\theta}\right], \\
& \forall \theta \in \mathcal{S}[s, T], \tau \in \mathcal{S}[\theta, T] .
\end{aligned}
$$

This means that

$$
\theta \mapsto V(\theta, X(\theta ; s, \xi))+\int_{s}^{\theta} g(r, X(r ; s, \xi)) d r
$$

is an $\mathbb{F}$-submartingale on $[s, T]$. Likewise, (3.6) implies that

Copyright $@$ by SIAM. Unauthorized reproduction of this article is prohibited. 


$$
\begin{aligned}
& V(\theta, X(\theta ; s, \xi))+\int_{s}^{\theta} g(r, X(r ; s, \xi)) d r \\
&=\mathbb{E}\left[V(\tau, X(\tau ; s, \xi))+\int_{s}^{\tau} g(r, X(r ; s, \xi)) d r \mid \mathcal{F}_{\theta}\right], \\
& \forall \theta \in \mathcal{S}[s, \bar{\tau}(s, \xi)], \tau \in \mathcal{S}[\theta, \bar{\tau}(s, \xi)],
\end{aligned}
$$

which means that

$$
\theta \mapsto V(\theta, X(\theta ; s, \xi))+\int_{s}^{\theta} g(r, X(r ; s, \xi)) d r
$$

is an $\mathbb{F}$-martingale on $[s, \bar{\tau}(s, \xi)]$.

Next, we would like to derive the HJB equation for value function $V(\cdot, \cdot)$. To this end, let us first make a convention: for any differentiable map $f: \mathbb{R}^{n} \rightarrow \mathbb{R}^{m}$, with $m>1$, gradient $f_{x}: \mathbb{R}^{n} \rightarrow \mathbb{R}^{m \times n}$, and for $m=1, f_{x}: \mathbb{R}^{n} \rightarrow \mathbb{R}^{n}$. Now, we recall a special case of Itô-Kunita's formula (see [15], [21]).

ThEOREM 3.2. Let $F:[0, T] \times \mathbb{R}^{n} \times \Omega \rightarrow \mathbb{R}$ satisfy the following:

(1) $(t, x) \mapsto F(t, x, \omega)$ is continuous a.s.;

(2) $x \mapsto F(t, x, \omega)$ is $C^{2}$ for each $t \in[0, T]$ a.s.;

(3) For each $x \in \mathbb{R}^{n}, t \mapsto F(t, x, \cdot)$ is a continuous semimartingale, with

$$
F(t, x)=F(0, x)+\int_{0}^{t} q^{0}(r, x) d r+\int_{0}^{t}\langle q(r, x), d W(r)\rangle, \quad(t, x) \in[0, T] \times \mathbb{R}^{n}
$$

for some $q^{0}(\cdot)$ and $q(\cdot)$ satisfying the following: For each $x \in \mathbb{R}^{n}, t \mapsto\left(q^{0}(t, x), q(t, x)\right)$ is $\mathbb{F}$-adapted, taking values in $\mathbb{R} \times \mathbb{R}^{d}$, and for almost all $(t, \omega) \in[0, T] \times \Omega, x \mapsto q(t, x)$ is $C^{1}$. Then

$$
\begin{aligned}
F(t, X(t))= & F(0, X(0))+\int_{0}^{t}\left\{q^{0}(r, X(r))+\left\langle b(r, X(r)), F_{x}(r, X(r))\right\rangle\right. \\
& \left.+\frac{1}{2} \operatorname{tr}\left[\sigma(r, X(r)) \sigma(r, X(r))^{T} F_{x x}(r, X(r))\right]+\operatorname{tr}\left[\sigma(r, X(r)) q_{x}(r, X(r))\right]\right\} d r \\
& +\int_{0}^{t}\left\langle q(r, X(r))+\sigma(r, X(r))^{T} F_{x}(r, X(r)), d W(r)\right\rangle .
\end{aligned}
$$

According to our convention, $q_{x}$ is taking values in $\mathbb{R}^{d \times n}$, and $F_{x}$ is taking values in $\mathbb{R}^{n}$. Now, for any $(s, \xi) \in \mathcal{D}^{p}$, suppose $\bar{\tau}(s, \xi)$ is the corresponding minimum optimal stopping time. Suppose value function $V(\cdot, \cdot)$ admits the following representation:

$$
V(t, x)=V(s, x)+\int_{s}^{t} q^{0}(r, x) d r+\int_{s}^{t}\langle q(r, x), d W(r)\rangle, \quad(t, x) \in[s, T] \times \mathbb{R}^{n},
$$

with $q^{0}(\cdot)$ and $q(\cdot)$ being undetermined. Then, by Itô-Kunita's formula, for any $t \in \mathcal{S}[s, T]$,

$$
\begin{aligned}
V(t, X(t ; s, \xi))=V( & s, \xi)+\int_{s}^{t}\left\{q^{0}(r, X(r ; s, \xi))+\left\langle b(r, X(r ; s, \xi)), V_{x}(r, X(r ; s, \xi))\right\rangle\right. \\
& +\frac{1}{2} \operatorname{tr}\left[\sigma(r, X(r ; s, \xi)) \sigma(r, X(r ; s, \xi))^{T} V_{x x}(r, X(r ; s, \xi))\right] \\
& \left.+\operatorname{tr}\left[\sigma(r, X(r ; s, \xi)) q_{x}(r, X(r ; s, \xi))\right]\right\} d r \\
& +\int_{s}^{t}\left\langle q(r, X(r ; s, \xi))+\sigma(r, X(r ; s, \xi))^{T} V_{x}(r, X(r ; s, \xi)), d W(r)\right\rangle .
\end{aligned}
$$


Hence, by (3.2), we have

$$
\begin{aligned}
0 \leq & \mathbb{E}\left[\int_{s}^{t} g(r, X(r ; s, \xi)) d r+V(t, X(t ; s, \xi))-V(s, \xi) \mid \mathcal{F}_{s}\right] \\
= & \mathbb{E}\left[\int _ { s } ^ { t } \left\{g(r, X(r ; s, \xi))+q^{0}(r, X(r ; s, \xi))+\left\langle b(r, X(r ; s, \xi)), V_{x}(r, X(r ; s, \xi))\right\rangle\right.\right. \\
& +\frac{1}{2} \operatorname{tr}\left[\sigma(r, X(r ; s, \xi)) \sigma(r, X(r ; s, \xi))^{T} V_{x x}(r, X(r ; s, \xi))\right] \\
& \left.\left.+\operatorname{tr}\left[\sigma(r, X(r ; s, \xi)) q_{x}(r, X(r ; s, \xi))\right]\right\} d r \mid \mathcal{F}_{s}\right] .
\end{aligned}
$$

Dividing it by $(t-s)$ and sending $t \rightarrow s$, we obtain

$$
\begin{aligned}
0 \leq & g(s, \xi)+q^{0}(s, \xi)+V_{x}(s, \xi) b(s, \xi)+\frac{1}{2} \operatorname{tr}\left[\sigma(s, \xi) \sigma(s, \xi)^{T} V_{x x}(s, \xi)\right] \\
& +\operatorname{tr}\left[\sigma(s, \xi) q_{x}(s, \xi)\right] \quad \text { a.s., } \quad \forall(s, \xi) \in \mathcal{D}^{p} .
\end{aligned}
$$

On the other hand, on the set $\{V(s, \xi)<h(s, \xi)\}$, one has $\bar{\tau}(s, \xi)>s$, and

$$
\theta \mapsto V(\theta, X(\theta ; s, \xi))+\int_{s}^{\theta} g(t, X(t ; s, \xi)) d t
$$

is a martingale on $[s, \bar{\tau}(s, \xi))$. Hence, it is necessary that

$$
\begin{aligned}
0= & g(s, \xi)+q^{0}(s, \xi)+\left\langle b(s, \xi), V_{x}(s, \xi)^{T}\right\rangle+\frac{1}{2} \operatorname{tr}\left[\sigma(s, \xi) \sigma(s, \xi)^{T} V_{x x}(s, \xi)\right] \\
& +\operatorname{tr}\left[\sigma(s, \xi) q_{x}(s, \xi)\right] \quad \text { a.s. on }\{V(s, \xi)<h(s, \xi)\}, \quad \forall(s, \xi) \in \mathcal{D}^{p} .
\end{aligned}
$$

Therefore, it is reasonable to require that

$$
\left\{\begin{aligned}
& q^{0}(r, x) \geq-\frac{1}{2} \operatorname{tr}\left[\sigma(r, x) \sigma(r, x)^{T} V_{x x}(r, x)\right]-\left\langle b(r, x), V_{x}(r, x)\right\rangle \\
&-\operatorname{tr}\left[\sigma(r, x) q_{x}(r, x)\right]-g(r, x) \quad \text { a.s. }, \quad(r, x) \in[0, T] \times \mathbb{R}^{n} \\
& q^{0}(r, x)=-\frac{1}{2} \operatorname{tr}\left[\sigma(r, x) \sigma(r, x)^{T} V_{x x}(r, x)\right]-\left\langle b(r, x), V_{x}(r, x)\right\rangle \\
&-\operatorname{tr}\left[\sigma(r, x) q_{x}(r, x)\right]-g(r, x) \quad \text { as on }\{V(r, x)<h(r, x)\}, \\
&(r, x) \in[0, T] \times \mathbb{R}^{n}
\end{aligned}\right.
$$

If we let $\beta: \mathbb{R} \rightarrow[0,+\infty]$ be a monotone graph defined by

$$
\beta(\rho)= \begin{cases}\phi, & \rho>0 \\ {[0,+\infty),} & \rho=0, \\ 0, & \rho<0,\end{cases}
$$

then we should have

$$
\begin{aligned}
q^{0}(r, x) \in-\frac{1}{2} \operatorname{tr}\left[\sigma(r, x) \sigma(r, x)^{T} V_{x x}(r, x)\right]-\left\langle b(r, x), V_{x}(r, x)\right\rangle-\operatorname{tr}\left[\sigma(r, x) q_{x}(r, x)\right] \\
+\beta(V(r, x)-h(r, x))-g(r, x), \quad \text { a.s. } \quad(r, x) \in[0, T] \times \mathbb{R}^{n},
\end{aligned}
$$

Copyright $@$ by SIAM. Unauthorized reproduction of this article is prohibited. 
which is understood as follows:

$$
\left\{\begin{array}{c}
q^{0}(r, x)=-\frac{1}{2} \operatorname{tr}\left[\sigma(r, x) \sigma(r, x)^{T} V_{x x}(r, x)\right]-\left\langle b(r, x), V_{x}(r, x)\right\rangle-\operatorname{tr}\left[\sigma(r, x) q_{x}(r, x)\right] \\
+\zeta(r, x)-g(r, x) \quad \text { a.s. } \quad(r, x) \in[0, T] \times \mathbb{R}^{n}, \\
\zeta(r, x) \in \beta(V(r, x)-h(r, x)) \quad \text { a.s. } \quad(r, x) \in[0, T] \times \mathbb{R}^{n} .
\end{array}\right.
$$

In the above, $\zeta(\cdot, x)$ is required to be $\mathbb{F}$-adapted. Consequently, we should have

$$
\left\{\begin{aligned}
V(t, x)= & h(T, x)+\int_{t}^{T}\left\{\frac{1}{2} \operatorname{tr}\left[\sigma(r, x) \sigma(r, x)^{T} V_{x x}(r, x)\right]+\left\langle b(r, x), V_{x}(r, x)\right\rangle\right. \\
& +\operatorname{tr}\left[\sigma(r, x) q_{x}(r, x)\right] \\
& -\zeta(r, x)+g(r, x)\} d r-\int_{t}^{T}\langle q(r, x), d W(r)\rangle, \quad t \in[0, T], \quad x \in \mathbb{R}^{n}, \\
\zeta(t, x) \in & \beta(V(t, x)-h(t, x)), \quad t \in[0, T], \quad x \in \mathbb{R}^{n} .
\end{aligned}\right.
$$

We call (3.20) a BSPDVI. Note that, in (3.20), the unknown is the triple of $\mathbb{F}$-adapted random fields $(V, q, \zeta):[0, T] \times \mathbb{R}^{n} \times \Omega \rightarrow \mathbb{R} \times \mathbb{R}^{d} \times \mathbb{R}$. Note that the last inclusion in (3.20) is equivalent to the following:

$$
\left\{\begin{array}{l}
V(t, x)-h(t, x) \leq 0, \quad \zeta(t, x) \geq 0, \quad(t, x) \in[0, T] \times \mathbb{R}^{n} \text { a.s. } \\
{[V(t, x)-h(t, x)] \zeta(t, x)=0,}
\end{array}\right.
$$

4. Adapted solutions. In this section, we will introduce notions of adapted solutions for BSPDVI (3.20) and will carry out some preliminary studies. To begin with, let us make a little preparation.

By a multi-index $\alpha$, we mean $\alpha=\left(\alpha_{1}, \ldots, \alpha_{n}\right)$, with each $\alpha_{i}$ being nonnegative integers, and we define $|\alpha|=\sum_{i=1}^{n} \alpha_{i}$. We write $x=\left(x_{1}, \ldots, x_{n}\right)$ for any generic point in $\mathbb{R}^{n}$. For any multi-index $\alpha \equiv\left(\alpha_{1}, \ldots, \alpha_{n}\right)$ and any smooth function $f(\cdot)$, denote

$$
\partial^{\alpha} f(x)=\partial_{x_{1}}^{\alpha_{1}} \cdots \partial_{x_{n}}^{\alpha_{n}} f(x) .
$$

For any domain $G \subseteq \mathbb{R}^{n}$ ( $G$ is allowed to be $\left.\mathbb{R}^{n}\right)$, let $C^{k}(G, \mathbb{R})$ be the set of all functions $f: G \rightarrow \mathbb{R}$ such that

$$
\sup _{x \in G,|\alpha| \leq k}\left|\partial^{\alpha} f(x)\right|<\infty
$$

We may similarly define spaces $C^{k}\left(G ; \mathbb{R}^{n}\right)$ and $C^{k}\left(G ; \mathbb{R}^{n \times d}\right)$, etc. Clearly, these are Banach spaces. Next, we let $W^{m, p}(G ; \mathbb{R})$ be the usual Sobolev space of all functions $f(\cdot)$ such that

$$
\|f(\cdot)\|_{W^{m, p}(G)}^{p} \equiv \sum_{|\alpha| \leq m} \int_{G}\left|\partial^{\alpha} f(x)\right|^{p} d x<\infty
$$

and $H^{m}\left(\mathbb{R}^{n}\right)=W^{m, 2}\left(\mathbb{R}^{n}\right)$. For any Banach space $B$, let $L_{\mathbb{F}}^{\infty}(0, T ; B)$ be the space of all bounded $\mathbb{F}$-progressively measurable maps $f:[0, T] \times \Omega \rightarrow B$, with the norm

$$
\|f(\cdot)\|_{L_{\mathbb{F}}^{\infty}(0, T ; B)}=\operatorname{esssup}_{(t, \omega) \in[0, T] \times \Omega}\|f(t, \omega)\|_{B} .
$$

Here, $B$ could be $C^{k}\left(G ; \mathbb{R}^{n}\right)$, say. Similarly, we let $C_{\mathbb{F}}(0, T ; B)$ be the space of all $B$-valued $\mathbb{F}$-adapted continuous processes, which is a closed subspace of $L_{\mathbb{F}}^{\infty}(0, T ; B)$.

We now introduce the following definition. 
Definition 4.1. (i) A triple of random fields $(V, q, \zeta)$ is called an adapted strong solution of (3.20) if for each $x \in \mathbb{R}^{n}, t \mapsto(V(t, x), q(t, x), \zeta(t, x))$ is $\mathbb{F}$-adapted and for almost all $(t, x, \omega) \in[0, T] \times \mathbb{R}^{n} \times \Omega, x \mapsto V(t, x, \omega)$ is twice differentiable, $x \mapsto q(t, x, \omega)$ is once differentiable such that (3.20) is satisfied for almost all $(x, \omega) \in \mathbb{R}^{n} \times \Omega$.

(ii) An adapted strong solution $(V, q, \zeta)$ of $(3.20)$ is called an adapted classical solution of (3.20) if for almost all $(t, \omega) \in[0, T] \times \Omega, x \mapsto V(t, x, \omega)$ is $C^{2}, x \mapsto$ $q(t, x, \omega)$ is $C^{1}$, and $x \mapsto \zeta(t, x)$ is continuous.

Once we have the well-posedness of our BSPDVI (which will be treated in the next section), it is natural to ask if solution $V(\cdot, \cdot)$ to the BSPDVI has anything to do with our Problem (S)? The following result answers this question: Under appropriate conditions, the solution of BSPDVI (3.20) coincides with the value function of Problem (S), via which an optimal stopping time can be identified.

TheOrem 4.2. Let $(\mathrm{H} 1)-(\mathrm{H} 2)$ hold. Suppose $(V, q, \zeta)$ is an adapted classical solution to BSPDVI (3.20). Then $V(\cdot, \cdot)$ is the value function of Problem (S). Consequently, part $V(\cdot, \cdot)$ of adapted classical solution $(V, q, \zeta)$ to $(3.20)$ is unique. Moreover, the following gives the smallest optimal stopping time of Problem (S):

$$
\bar{\tau}(s, \xi)=\inf \{t \in[s, T] \mid V(t, X(t ; s, \xi))=h(t, X(t ; s, \xi))\} .
$$

Proof. Let $(s, \xi) \in \mathcal{D}^{p}$, and define $\bar{\tau}(s, \xi)$ by (4.5). By the Itô-Kunita formula, together with BSPDVI (3.20), we have

$$
V(s, \xi)=\mathbb{E}\left[\int_{s}^{\tau} g(r, X(r ; s, \xi)) d r+V(\tau, X(\tau ; s, \xi)) \mid \mathcal{F}_{s}\right] \quad \forall \tau \in \mathcal{S}[s, \bar{\tau}(s, \xi)] .
$$

Hence, taking $\tau=\bar{\tau}(s, \xi)$, we have

$$
\begin{aligned}
V(s, \xi) & =\mathbb{E}\left[V(\bar{\tau}(s, \xi), X(\bar{\tau}(s, \xi) ; s, \xi))+\int_{s}^{\bar{\tau}(s, \xi)} g(r, X(r ; s, \xi)) d r \mid \mathcal{F}_{s}\right] \\
& =\mathbb{E}\left[h(\bar{\tau}(s, \xi), X(\bar{\tau}(s, \xi) ; s, \xi))+\int_{s}^{\bar{\tau}(s, \xi)} g(r, X(r ; s, \xi)) d r \mid \mathcal{F}_{s}\right]=J_{s, \xi}(\bar{\tau}(s, \xi)) .
\end{aligned}
$$

This means that $\bar{\tau}(s, \xi)$ is an optimal stopping time for our Problem (S). From the above, we further conclude that part $V(\cdot, \cdot)$ of adapted solution $(V, q, \zeta)$ to BSPDVI (3.20) is unique, and from $(4.5), \bar{\tau}(s, \xi)$ has to be the smallest optimal stopping time (noting (ii) of Theorem 3.1).

Next, we would like to make a reduction which will be very useful below. To this end, let

$$
h(t, x)=h(0, x)+\int_{0}^{t} \mu^{0}(r, x) d r+\int_{0}^{t}\langle\mu(r, x), d W(r)\rangle, \quad t \in[0, T]
$$

for some suitable $\mu^{0}(\cdot)$ and $\mu(\cdot)$. Suppose $(V, q, \zeta)$ is an adapted classical solution to BSPDVI (3.20), and all the coefficients have required order of derivatives. We fix $p \geq 2$, and let

$$
\left\{\begin{array}{l}
\bar{V}(t, x)=\frac{V(t, x)-h(t, x)}{1+|x|^{p}}, \\
\bar{q}(t, x)=\frac{q(t, x)-\mu(t, x)}{1+|x|^{p}} . \\
\bar{\zeta}(t, x)=\frac{\zeta(t, x)}{1+|x|^{p}} .
\end{array} \quad(t, x) \in[0, T] \times \mathbb{R}^{n} \quad\right. \text { a.s. }
$$

Copyright (c) by SIAM. Unauthorized reproduction of this article is prohibited. 
Note that, in the case $x \mapsto V(t, x)-h(t, x)$ grows at most linearly, $x \mapsto \bar{V}(t, x)$ will be $L^{2}$-integrable over $\mathbb{R}^{n}$. According to the above, one has

$$
\left\{\begin{aligned}
V(t, x) & =\left(1+|x|^{p}\right) \bar{V}(t, x)+h(t, x), \\
q(t, x) & =\left(1+|x|^{p}\right) \bar{q}(t, x)+\mu(t, x), \\
\zeta(t, x) & =\left(1+|x|^{p}\right) \bar{\zeta}(t, x) .
\end{aligned}\right.
$$

Consequently (suppressing $(t, x)$ ),

$$
\left\{\begin{aligned}
V_{x}= & \left(1+|x|^{p}\right) \bar{V}_{x}+p|x|^{p-2} \bar{V} x+h_{x}, \\
V_{x x}= & \left(1+|x|^{p}\right) \bar{V}_{x x}+p|x|^{p-2}\left[\left(x \bar{V}_{x}\right)^{T}+x \bar{V}_{x}\right] \\
& +\left[p(p-2)|x|^{p-4} x x^{T}+p|x|^{p-2} I\right] \bar{V}+h_{x x}, \\
q_{x}= & \left(1+|x|^{p}\right) \bar{q}_{x}+p|x|^{p-2} \bar{q} x^{T}+\mu_{x} .
\end{aligned}\right.
$$

Hence,

$$
\begin{aligned}
& \bar{V}(t, x) \equiv\left(1+|x|^{p}\right)^{-1}[V(t, x)-h(t, x)] \\
& =\left(1+|x|^{p}\right)^{-1}\left(h(T, x)-h(t, x)+\int_{t}^{T}\left\{\frac{1}{2} \operatorname{tr}\left[\sigma(r, x) \sigma(r, x)^{T} V_{x x}(r, x)\right]\right.\right. \\
& \left.+\left\langle b(r, x), V_{x}(r, x)\right\rangle+\operatorname{tr}\left[\sigma(r, x) q_{x}(r, x)\right]-\zeta(r, x)+g(r, x)\right\} d r \\
& \left.-\int_{t}^{T}\langle q(r, x), d W(r)\rangle\right) \\
& =\int_{t}^{T} \frac{\mu^{0}(r, x)}{1+|x|^{p}} d r+\int_{t}^{T}\left\langle\frac{\mu(r, x)}{1+|x|^{p}}, d W(r)\right\rangle+\int_{t}^{T}\left\{\frac { 1 } { 2 } \operatorname { t r } \left[\sigma(r, x) \sigma(r, x)^{T}\right.\right. \\
& \left.\cdot\left(\bar{V}_{x x}+\frac{p|x|^{p-2}\left[x \bar{V}_{x}^{T}+\bar{V}_{x} x^{T}\right]}{1+|x|^{p}}+\frac{p|x|^{p-4}\left[(p-2) x x^{T}+|x|^{2} I\right] \bar{V}+h_{x x}}{1+|x|^{p}}\right)\right] \\
& +\left\langle b(r, x), \bar{V}_{x}+\frac{p|x|^{p-2} \bar{V} x+h_{x}}{1+|x|^{p}}\right\rangle+\operatorname{tr}\left[\sigma(r, x)\left(\bar{q}_{x}+\frac{p|x|^{p-2} \bar{q} x^{T}+\mu_{x}}{1+|x|^{p}}\right)\right] \\
& \left.-\frac{\zeta(r, x)-g(r, x)}{1+|x|^{p}}\right\} d r-\int_{t}^{T}\left\langle\frac{q(r, x)}{1+|x|^{p}}, d W(s)\right\rangle \\
& =\int_{t}^{T}\left\{\frac{1}{2} \operatorname{tr}\left[\sigma(r, x) \sigma(r, x)^{T} \bar{V}_{x x}\right]+\left\langle b(r, x)+\frac{p|x|^{p-2} \sigma(r, x) \sigma(r, x)^{T} x}{1+|x|^{p}}, \bar{V}_{x}\right\rangle\right. \\
& +\frac{p|x|^{p-4}\left[(p-2)\left|\sigma(r, x)^{T} x\right|^{2}+|x|^{2}|\sigma(r, x)|^{2}\right]+2 p|x|^{p-2}\langle b(r, x), x\rangle}{2\left(1+|x|^{p}\right)} \bar{V} \\
& +\operatorname{tr}\left[\sigma(r, x) \bar{q}_{x}\right]+\left\langle\frac{p|x|^{p-2} \sigma(r, x)^{T} x}{1+|x|^{p}}, \bar{q}\right\rangle-\bar{\zeta} \\
& +\left(1+|x|^{p}\right)^{-1}\left[\frac{1}{2} \operatorname{tr}\left(\sigma(r, x) \sigma(r, x)^{T} h_{x x}(r, x)\right)+\left\langle b(r, x), h_{x}(r, x)\right\rangle\right. \\
& \left.\left.+\operatorname{tr}\left(\sigma(r, x) \mu_{x}(r, x)\right)+\mu^{0}(r, x)+g(r, x)\right]\right\} d r-\int_{s}^{t}\langle\bar{q}(r, x), d W(s)\rangle \\
& \equiv \int_{s}^{t}\left\{\frac{1}{2} \operatorname{tr}\left[\sigma(r, x) \sigma(r, x)^{T} \bar{V}_{x x}(r, x)\right]+\left\langle\widetilde{b}(r, x), \bar{V}_{x}(r, x)\right\rangle+\widetilde{b}^{0}(r, x) \bar{V}(r, x)\right. \\
& \left.+\operatorname{tr}\left[\sigma(r, x) \bar{q}_{x}(r, x)\right]+\left\langle\widetilde{\sigma}^{0}(r, x), \bar{q}(r, x)\right\rangle-\bar{\zeta}(r, x)+\widetilde{g}(r, x)\right\} d r \\
& -\int_{s}^{t}\langle\bar{q}(r, x), d W(s)\rangle
\end{aligned}
$$

Copyright $@$ by SIAM. Unauthorized reproduction of this article is prohibited. 
with

(4.13)

$$
\left\{\begin{aligned}
\widetilde{b}(r, x) & =b(r, x)+\frac{p|x|^{p-2} \sigma(r, x) \sigma(r, x)^{T} x}{1+|x|^{p}} \\
\widetilde{b}^{0}(r, x) & =\frac{p|x|^{p-4}\left[(p-2)\left|\sigma(r, x)^{T} x\right|^{2}+|x|^{2}|\sigma(r, x)|^{2}\right]+2 p|x|^{p-2}\langle b(r, x), x\rangle}{2\left(1+|x|^{p}\right)} \\
\widetilde{\sigma}^{0}(r, x)= & \frac{p|x|^{p-2} \sigma(r, x)^{T} x}{1+|x|^{p}} \\
\widetilde{g}(r, x)= & \left(1+|x|^{p}\right)^{-1}\left[\frac{1}{2} \operatorname{tr}\left(\sigma(r, x) \sigma(r, x)^{T} h_{x x}(r, x)\right)+\left\langle b(r, x), h_{x}(r, x)\right\rangle\right. \\
& \left.+\operatorname{tr}\left(\sigma(r, x) \mu_{x}(r, x)\right)+\mu^{0}(r, x)+g(r, x)\right] .
\end{aligned}\right.
$$

Note that, by the definition of $\beta$, we see that

$$
\zeta(t, x) \in \beta(V(t, x)-h(t, x)), \quad(t, x) \in[0, T] \times \mathbb{R}^{n} \quad \text { a.s. }
$$

is equivalent to

$$
\bar{\zeta}(t, x) \in \beta(\bar{V}(t, x)), \quad(t, x) \in[0, T] \times \mathbb{R}^{n} \quad \text { a.s. }
$$

Next, we have

$$
\left\{\begin{array}{l}
\operatorname{tr}\left(\sigma \sigma^{T} \bar{V}_{x x}\right)=\nabla \cdot\left(\sigma \sigma^{T} \bar{V}_{x}\right)-\left\langle\nabla \cdot\left(\sigma \sigma^{T}\right), \bar{V}_{x}\right\rangle, \\
\operatorname{tr}\left(\sigma \bar{q}_{x}\right)=\nabla \cdot(\sigma \bar{q})-\langle\nabla \cdot \sigma, \bar{q}\rangle
\end{array}\right.
$$

where (with $\sigma=\left(\sigma_{1}, \ldots, \sigma_{d}\right)$, each $\sigma_{i}$ takes values in $\mathbb{R}^{n}$ )

$$
\nabla \cdot \sigma=\left(\nabla \cdot \sigma_{1}, \ldots, \nabla \cdot \sigma_{d}\right)^{T}
$$

and

$$
\begin{aligned}
& \nabla \cdot\left(\sigma \sigma^{T}\right)=\sum_{k=1}^{d} \nabla \cdot\left(\sigma_{k} \sigma_{k}^{T}\right)=\sum_{k=1}^{d}\left(\nabla \cdot\left(\sigma_{1 k} \sigma_{k}\right), \ldots, \nabla \cdot\left(\sigma_{n k} \sigma_{k}\right)\right)^{T} \\
& =\sum_{k=1}^{d}\left(\sigma_{1 k}\left(\nabla \cdot \sigma_{k}\right), \ldots, \sigma_{n k}\left(\nabla \cdot \sigma_{k}\right)\right)^{T}+\sum_{k=1}^{d}\left(\sigma_{k}^{T}\left(\sigma_{1 k}\right)_{x}, \ldots, \sigma_{k}^{T}\left(\sigma_{n k}\right)_{x}\right)^{T} \\
& =\sum_{k=1}^{d}\left(\nabla \cdot \sigma_{k}\right) \sigma_{k}+\sum_{k=1}^{d}\left(\sigma_{k}\right)_{x} \sigma_{k}=\sum_{k=1}^{d}\left[\left(\nabla \cdot \sigma_{k}\right) I+\left(\sigma_{k}\right)_{x}\right] \sigma_{k} .
\end{aligned}
$$

Then, we can get

$$
\begin{aligned}
& \frac{1}{2} \operatorname{tr}\left(\sigma \sigma^{T} \bar{V}_{x x}\right)+\left\langle\widetilde{b}, \bar{V}_{x}\right\rangle+\widetilde{b}^{0} \bar{V}+\operatorname{tr}\left(\sigma \bar{q}_{x}\right)+\left\langle\widetilde{\sigma}^{0}, \bar{q}\right\rangle-\bar{\zeta}+\widetilde{g} \\
& =\frac{1}{2} \nabla \cdot\left(\sigma \sigma^{T} \bar{V}_{x}\right)+\left\langle\widetilde{b}-\nabla \cdot\left(\sigma \sigma^{T}\right), \bar{V}_{x}\right\rangle+\widetilde{b}^{0} \bar{V}+\nabla \cdot(\sigma \bar{q})+\left\langle\widetilde{\sigma}^{0}-\nabla \cdot \sigma, \bar{q}\right\rangle-\bar{\zeta}+\bar{g} .
\end{aligned}
$$

According to the above reduction, we have the following divergence form of our 
BSPDVI:

$$
\left\{\begin{aligned}
\bar{V}(t, x)= & \int_{t}^{T}\left\{\frac{1}{2} \nabla \cdot\left[\sigma(r, x) \sigma(r, x)^{T} \bar{V}_{x}(r, x)\right]+\left\langle\bar{b}(r, x), \bar{V}_{x}(r, x)\right\rangle+\bar{b}^{0}(r, x) \bar{V}(r, x)\right. \\
& \left.+\nabla \cdot[\sigma(r, x) \bar{q}(r, x)]+\left\langle\bar{\sigma}^{0}(r, x), \bar{q}(r, x)\right\rangle-\bar{\zeta}(r, x)+\bar{g}(r, x)\right\} d r \\
& -\int_{t}^{T}\langle\bar{q}(r, x), d W(r)\rangle, \quad t \in[0, T], \quad x \in \mathbb{R}^{n}, \\
\bar{\zeta}(t, x) \in & \beta(\bar{V}(t, x)), \quad t \in[0, T], \quad x \in \mathbb{R}^{n},
\end{aligned}\right.
$$

with

$$
\left\{\begin{aligned}
\bar{b}(r, x)= & \widetilde{b}(r, x)-\nabla \cdot\left[\sigma(r, x) \sigma(r, x)^{T}\right] \\
= & b(r, x)-\nabla \cdot\left[\sigma(r, x) \sigma(r, x)^{T}\right]+\frac{p|x|^{p-2} \sigma(r, x) \sigma(r, x)^{T} x}{1+|x|^{p}}, \\
\bar{b}^{0}(r, x)= & \widetilde{b}^{0}(r, x) \\
= & \frac{p|x|^{p-4}\left[(p-2)\left|\sigma(r, x)^{T} x\right|^{2}+|x|^{2}|\sigma(r, x)|^{2}\right]+2 p|x|^{p-2}\langle b(r, x), x\rangle}{2\left(1+|x|^{p}\right)}, \\
\bar{\sigma}^{0}(r, x)= & \widetilde{\sigma}^{0}(r, x)-\nabla \cdot \sigma(r, x)=\frac{p|x|^{p-2} \sigma(r, x)^{T} x}{1+|x|^{p}}-\nabla \cdot \sigma(r, x), \\
\bar{g}(r, x)= & \widetilde{g}(r, x)=\left(1+|x|^{p}\right)^{-1}\left[\frac{1}{2} \operatorname{tr}\left(\sigma(r, x) \sigma(r, x)^{T} h_{x x}(r, x)\right)+\left\langle b(r, x), h_{x}(r, x)\right\rangle\right. \\
& \left.+\operatorname{tr}\left(\sigma(r, x) \mu_{x}(r, x)\right)+\mu^{0}(r, x)+g(r, x)\right] .
\end{aligned}\right.
$$

In order for the above reduction to be possible and for the purpose of some other further discussions, we introduce the following assumption.

Assumption (H3). For some $k>2+\frac{n}{2}$, maps $b(\cdot), \sigma(\cdot), g(\cdot), \mu_{0}(\cdot), \mu(\cdot)$, and $h(0, \cdot)$ satisfy the following:

$$
\left\{\begin{array}{l}
b(\cdot) \in L_{\mathbb{F}}^{\infty}\left(0, T ; C^{k}\left(\mathbb{R}^{n} ; \mathbb{R}^{n}\right)\right), \quad \sigma(\cdot), \mu(\cdot) \in L_{\mathbb{F}}^{\infty}\left(0, T ; C^{k}\left(\mathbb{R}^{n} ; \mathbb{R}^{n \times d}\right)\right), \\
g(\cdot), \mu_{0}(\cdot) \in L_{\mathbb{F}}^{\infty}\left(0, T ; C^{k}\left(\mathbb{R}^{n} ; \mathbb{R}\right)\right), \quad h(0, \cdot) \in C^{k}\left(\mathbb{R}^{n} ; \mathbb{R}\right) .
\end{array}\right.
$$

Under (H3), we see that

$$
\left\{\begin{array}{l}
|\bar{b}(t, x)|+\left|\bar{b}^{0}(t, x)\right|+\left|\bar{\sigma}^{0}(t, x)\right| \leq C, \quad(t, x) \in(t, x) \in[0, T] \times \mathbb{R}^{n} \quad \text { a.s. } \\
|\bar{g}(t, x)| \leq C\left(1+|x|^{p}\right)^{-1},
\end{array}\right.
$$

In what follows, we will choose $p>2$, which will lead to

$$
\int_{\mathbb{R}^{n}}|\bar{g}(t, x)| d x \leq C \quad \forall t \in[0, T] \text { a.s. }
$$

We may introduce adapted classical and strong solutions to the divergence form of BSPDVI (4.20), similar to Definition 4.1. On the other hand, let us now introduce the following notion.

Definition 4.3. A triple

$$
(\bar{V}, \bar{q}, \bar{\zeta}) \in L_{\mathbb{F}}^{2}\left(0, T ; H^{1}\left(\mathbb{R}^{n}\right)\right) \times L_{\mathbb{F}}^{2}\left(0, T ; L^{2}\left(\mathbb{R}^{n} ; \mathbb{R}^{d}\right)\right) \times L_{\mathbb{F}}^{2}\left(0, T ; L^{2}\left(\mathbb{R}^{n}\right)\right)
$$

Copyright (c) by SIAM. Unauthorized reproduction of this article is prohibited. 
is called an adapted weak solution of (4.20) if, for any $\varphi \in H^{1}\left(\mathbb{R}^{n}\right)$,

$$
\begin{aligned}
& \int_{\mathbb{R}^{n}} \bar{V}(t, x) \varphi(x) d x=\int_{t}^{T} \int_{\mathbb{R}^{n}}\left\{-\left\langle\frac{1}{2} \sigma(r, x)^{T} \bar{V}_{x}(r, x)+\bar{q}(r, x), \sigma(r, x)^{T} \varphi_{x}(x)\right\rangle\right. \\
& +\left[\left\langle\bar{b}(r, x), \bar{V}_{x}(r, x)\right\rangle+\bar{b}^{0}(r, x) \bar{V}(r, x)\right. \\
& \left.\left.+\left\langle\bar{\sigma}^{0}(r, x), \bar{q}(r, x)\right\rangle+\bar{g}(r, x)-\bar{\zeta}(r, x)\right] \varphi(x)\right\} d x d r \\
& -\int_{t}^{T}\left\langle\int_{\mathbb{R}^{n}} \bar{q}(r, x) \varphi(x) d x, d W(r)\right\rangle, \quad t \in[0, T],
\end{aligned}
$$

and

$$
\begin{cases}\bar{V}(t, x) \leq 0, \quad \bar{\zeta}(t, x) \geq 0, & (t, x) \in[0, T] \times \mathbb{R}^{n} \quad \text { a.s. }, \\ \bar{V}(t, x) \bar{\zeta}(t, x)=0, & \text { a.e. }(t, x) \in[0, T] \times \mathbb{R}^{n} \quad \text { a.s. }\end{cases}
$$

We point out that any adapted strong solution $(\bar{V}, \bar{q}, \bar{\zeta})$ of $(4.20)$ must be an adapted weak solution of (4.20). Similar to [16], we can show, by an argument using integration by parts, that an adapted weak solution is an adapted strong (classical) solution if it has the regularity that the latter requires.

5. Well-posedness of the BSPDVI. In this section, we are going to discuss the issue of the well-posedness of BSPVDI. First, we have the following.

TheOrEm 5.1. Suppose (H3) holds. Let $(\bar{V}, \bar{q}, \bar{\zeta})$ and $(\widetilde{V}, \widetilde{q}, \widetilde{\zeta})$ be adapted weak solutions to (4.20), with

$$
\left\{\begin{array}{l}
\bar{V}, \widetilde{V} \in L_{\mathbb{F}}^{2}\left(0, T ; H^{1}\left(\mathbb{R}^{n}\right)\right), \\
\bar{q}, \widetilde{q} \in L_{\mathbb{F}}^{2}\left(0, T ; L^{2}\left(\mathbb{R}^{n} ; \mathbb{R}^{d}\right)\right), \\
\bar{\zeta}, \widetilde{\zeta} \in L_{\mathbb{F}}^{2}\left(0, T ; L^{2}\left(\mathbb{R}^{n}\right)\right)
\end{array}\right.
$$

Then

$\bar{V}(t, x)=\widetilde{V}(t, x), \quad \bar{q}(t, x)=\widetilde{q}(t, x), \quad \bar{\zeta}(t, x)=\widetilde{\zeta}(t, x), \quad(t, x) \in[0, T] \times \mathbb{R}^{n}$, a.s.

Proof. Suppose $(\bar{V}, \bar{q}, \bar{\zeta})$ and $(\widetilde{V}, \widetilde{q}, \widetilde{\zeta})$ are two adapted weak solutions to BSPDVI (4.20). Set

$$
\widehat{V}=\bar{V}-\widetilde{V}, \quad \widehat{q}=\bar{q}-\widetilde{q}, \quad \widehat{\zeta}=\bar{\zeta}-\widetilde{\zeta}
$$

Then $(\widehat{V}, \widehat{q}, \widehat{\zeta})$ is an adapted weak solution to the following linear BSPDE:

$$
\begin{aligned}
\widehat{V}(t, x)= & \int_{t}^{T}\left\{\frac{1}{2} \nabla \cdot\left[\sigma(r, x) \sigma(r, x)^{T} \widehat{V}_{x}(r, x)\right]+\left\langle\bar{b}(r, x), \widehat{V}_{x}(r, x)\right\rangle+\bar{b}^{0}(r, x) \widehat{V}(r, x)\right. \\
& \left.+\nabla \cdot[\sigma(r, x) \widehat{q}(r, x)]+\left\langle\bar{\sigma}^{0}(r, x), \widehat{q}(r, x)\right\rangle-\widehat{\zeta}(r, x)\right\} d r \\
& -\int_{t}^{T}\langle\widehat{q}(r, x), d W(r)\rangle, \quad t \in[0, T], \quad x \in \mathbb{R}^{n} .
\end{aligned}
$$


By Itô's type formula (see [16] for details), we have

$$
\begin{array}{r}
=\mathbb{E} \int_{t}^{T} \int_{\mathbb{R}^{n}}\left\{-\left|\sigma^{T} \widehat{V}_{x}+\widehat{q}-\bar{\sigma}^{0} \widehat{V}\right|^{2}+\left|\bar{\sigma}^{0}\right|^{2} \widehat{V}^{2}\right. \\
\left.\quad-2\left\langle\sigma^{T} \widehat{V}_{x}, \bar{\sigma}^{0} \widehat{V}\right\rangle+\left[2 \bar{b}^{0}-(\nabla \cdot \bar{b})\right] \widehat{V}^{2}-2 \widehat{\zeta} \widehat{V}\right\} d x d r \\
=\mathbb{E} \int_{t}^{T} \int_{\mathbb{R}^{n}}\left\{-\left|\sigma^{T} \widehat{V}_{x}+\widehat{q}-\bar{\sigma}^{0} \widehat{V}\right|^{2}\right. \\
\left.+\left[\left|\bar{\sigma}^{0}\right|^{2}+\nabla \cdot\left(\sigma \bar{\sigma}^{0}\right)+2 \bar{b}^{0}-(\nabla \cdot \bar{b})\right] \widehat{V}^{2}-2 \widehat{\zeta} \widehat{V}\right\} d x d r
\end{array}
$$$$
\mathbb{E} \int_{\mathbb{R}^{n}}|\widehat{V}(t, x)|^{2} d x=\mathbb{E} \int_{t}^{T} \int_{\mathbb{R}^{n}}\left\{-\left|\sigma^{T} \widehat{V}_{x}\right|^{2}+\left[2 \bar{b}^{0}-(\nabla \cdot \bar{b})\right] \widehat{V}^{2}-2\left\langle\widehat{q}, \sigma^{T} \widehat{V}_{x}\right\rangle\right.
$$$$
\left.+2\left\langle\bar{\sigma}^{0}, \widehat{q}\right\rangle \widehat{V}-2 \widehat{\zeta V}-|\widehat{q}|^{2}\right\} d x d r
$$

Note that

$$
\begin{aligned}
\widehat{V}(t, x) \widehat{\zeta}(t, x) & \equiv[\bar{V}(t, x)-\widetilde{V}(t, x)][\bar{\zeta}(t, x)-\widetilde{\zeta}(t, x)] \\
& =-\bar{V}(t, x) \widetilde{\zeta}(t, x)-\widetilde{V}(t, x) \bar{\zeta}(t, x) \geq 0 .
\end{aligned}
$$

Thus, the above implies that

$$
\mathbb{E} \int_{\mathbb{R}^{n}}|\bar{V}(t, x)-\widetilde{V}(t, x)|^{2} d x \leq C \mathbb{E} \int_{t}^{T} \int_{\mathbb{R}^{n}}|\bar{V}(r, x)-\widetilde{V}(r, x)|^{2} d x d r .
$$

Hence, by Gronwall's inequality, we obtain that

$$
\bar{V}(t, x)=\widetilde{V}(t, x), \quad(t, x) \in[0, T] \times \mathbb{R}^{n}, \text { a.s. }
$$

Further, (5.5) implies that

$$
\bar{q}(t, x)=\widetilde{q}(t, x), \quad(t, x) \in[0, T] \times \mathbb{R}^{n}, \text { a.s. }
$$

Then, by virtue of (5.4), we have

$$
\bar{\zeta}(t, x)=\widetilde{\zeta}(t, x), \quad(t, x) \in[0, T] \times \mathbb{R}^{n}, \text { a.s. }
$$

which proves our conclusion.

To establish the existence of an adapted weak solution, we define

$$
\eta(\rho)= \begin{cases}0, & \rho \in(-\infty, 0] \bigcup(2, \infty), \\ \rho, & \rho \in(0,1], \\ 2-\rho, & \rho \in(1,2]\end{cases}
$$

and define

$$
\psi(\rho)=\int_{0}^{\rho} \int_{0}^{\tau} \eta(r) d r d \tau=\int_{0}^{\rho}(\rho-r) \eta(r) d r, \quad \rho \in \mathbb{R} .
$$

Thus, $\psi: \mathbb{R} \rightarrow \mathbb{R}$ is $C^{2}$, nondecreasing, and convex. 
Now, for any $\varepsilon>0$, we consider the following semilinear BSPDE:

$$
\begin{aligned}
V^{\varepsilon}(t, x)= & \int_{t}^{T}\left\{\frac{1}{2} \nabla \cdot\left(\sigma \sigma^{T} V_{x}^{\varepsilon}\right)+\left\langle\bar{b}, V_{x}^{\varepsilon}\right\rangle+\bar{b}^{0} V^{\varepsilon}+\nabla \cdot\left(\sigma q^{\varepsilon}\right)+\left\langle\bar{\sigma}^{0}, q^{\varepsilon}\right\rangle\right. \\
& \left.+\bar{g}-\psi\left(\frac{V^{\varepsilon}}{\varepsilon}\right)\right\} d r-\int_{t}^{T}\left\langle q^{\varepsilon}, d W(r)\right\rangle, \quad(t, x) \in[0, T] \times \mathbb{R}^{n} .
\end{aligned}
$$

The unknown of semilinear BSPDE (5.13) is the pair $\left(V^{\varepsilon}, q^{\varepsilon}\right)$ of $\mathbb{F}$-adapted random fields. The following is a special case of a relevant result found in [26].

TheOREM 5.2. Let (H3) hold. Then, semilinear BSPDE (5.13) admits unique adapted classical solution $\left(V^{\varepsilon}, q^{\varepsilon}\right)$. Moreover, for any $p>1$ and any compact set $K \subseteq \mathbb{R}^{n}$

$$
\mathbb{E}\left[\sup _{t \in[0, T], x \in K}\left|\partial^{\alpha} V^{\varepsilon}(t, x)\right|^{p}\right]+\mathbb{E} \int_{0}^{T} \sup _{x \in K}\left|\partial^{\alpha} q^{\varepsilon}(t, x)\right|^{p} d t<\infty \quad \forall|\alpha| \leq 2
$$

We hope that unique adapted classical solution $\left(V^{\varepsilon}, q^{\varepsilon}\right)$ of $(4.20)$ will converge to $(\bar{V}, \bar{q})$ in some sense, where $(\bar{V}, \bar{q}, \bar{\zeta})$ is the adapted weak solution to our BSPDVI (4.20). Moreover, it is a hope that value function $V$ of Problem (S) can be identified by $\bar{V}$ through (4.10). However, we note that, in the above estimate (5.14), the bound of the left-hand side depends not only on compact set $K$, but also depends on $\varepsilon>0$, in general. Hence, we first would like to establish some estimates for $\left(V^{\varepsilon}, q^{\varepsilon}\right)$ (on the whole space $[0, T] \times \mathbb{R}^{n}$ ), which are uniform in $\varepsilon>0$. To this end, we begin with several lemmas whose technical proofs will be given in the appendix.

Lemma 5.3. Let $\theta: \mathbb{R} \rightarrow[0, \infty)$ be convex and piecewise smooth. Suppose that

$$
0=\theta(0)=\min _{\rho \in \mathbb{R}} \theta(\rho)
$$

and

$$
\left[\theta^{\prime}(\rho)\right]^{2} \leq C \theta(\rho) \theta^{\prime \prime}(\rho) \quad \text { a.e. } \rho \in \mathbb{R}
$$

for some constant $C>0$. Let $\left(V^{\varepsilon}, q^{\varepsilon}\right)$ be the adapted classical solution to BSPDE (5.13). Then

$$
\begin{array}{r}
\mathbb{E} \int_{\mathbb{R}^{n}} \theta\left(V^{\varepsilon}(t, x)\right) d x+\mathbb{E} \int_{t}^{T} \int_{\mathbb{R}^{n}}\left\{\theta^{\prime \prime}\left(V^{\varepsilon}(r, x)\right)\left|\sigma(r, x)^{T} V_{x}^{\varepsilon}(r, x)+q^{\varepsilon}(r, x)\right|^{2}\right. \\
\left.+\theta^{\prime}\left(V^{\varepsilon}(r, x)\right) \psi\left(\frac{V^{\varepsilon}(r, x)}{\varepsilon}\right)\right\} d x d r \leq C \mathbb{E} \int_{t}^{T} \int_{\mathbb{R}^{n}}\left|\theta^{\prime}\left(V^{\varepsilon}(r, x)\right)\right| \\
\quad\left(\left|V^{\varepsilon}(r, x)\right|+|\bar{g}(r, x)|\right) d x d r, \quad t \in[0, T] .
\end{array}
$$

The above lemma can be used to establish several interesting estimates for $\left(V^{\varepsilon}, q^{\varepsilon}\right)$. LEMma 5.4. Let (H3) hold and $\left(V^{\varepsilon}, q^{\varepsilon}\right)$ be the classical solution to BSPDE (5.13). Then there exists a constant $C>0$, independent of $m \geq 1$ and $\varepsilon>0$ such 
that

$$
\begin{aligned}
& \sup _{t \in[0, T]} \mathbb{E} \int_{\mathbb{R}^{n}}\left|V^{\varepsilon}(t, x)\right|^{2 m} d x+\mathbb{E} \int_{0}^{T} \int_{\mathbb{R}^{n}} m^{2}\left|V^{\varepsilon}(r, x)\right|^{2 m-2}\left[\left|\sigma(r, x)^{T} V_{x}^{\varepsilon}(r, x)+q^{\varepsilon}(r, x)\right|^{2}\right. \\
& \left.\quad+m V^{\varepsilon}(r, x) \psi\left(\frac{V^{\varepsilon}(r, x)}{\varepsilon}\right)\right] d x d r \leq C e^{C m} \mathbb{E} \int_{0}^{T} \int_{\mathbb{R}^{n}}|\bar{g}(r, x)|^{2 m} d x d r, \quad \forall \varepsilon>0, m \geq 1, \\
& (5.18) \\
& \sup _{t \in[0, T]} \mathbb{E} \int_{\mathbb{R}^{n}}\left(V^{\varepsilon}(t, x)^{+}\right)^{2} d x+\mathbb{E} \int_{0}^{T} \int_{\mathbb{R}^{n}}\left\{\left|\sigma(r, x)^{T} V_{x}^{\varepsilon}(r, x)+q^{\varepsilon}(r, x)\right|^{2} I_{\left\{V^{\varepsilon}>0\right\}}\right.
\end{aligned}
$$$$
\left.+V^{\varepsilon}(r, x)^{+} \psi\left(\frac{V^{\varepsilon}(r, x)}{\varepsilon}\right)\right\} d x d r \leq C \mathbb{E} \int_{0}^{T} \int_{\mathbb{R}^{n}}|\bar{g}(r, x)|^{2} I_{\left\{V^{\varepsilon}>0\right\}} d x d r, \quad \forall \varepsilon>0,
$$

$$
\begin{aligned}
\sup _{t \in[0, T]} & \mathbb{E} \int_{\mathbb{R}^{n}} V^{\varepsilon}(t, x) \psi\left(\frac{V^{\varepsilon}(t, x)}{\varepsilon}\right) d x \\
& +\mathbb{E} \int_{0}^{T} \int_{\mathbb{R}^{n}} \psi\left(\frac{V^{\varepsilon}(r, x)}{\varepsilon}\right)^{2} d x d r \leq C \mathbb{E} \int_{0}^{T} \int_{\mathbb{R}^{n}}|\bar{g}(r, x)|^{2} d x d r .
\end{aligned}
$$

Next, differentiating BSPDE (5.13) with respect to $x_{k}$, we get

$$
\begin{aligned}
V_{x_{k}}^{\varepsilon}(t, x)= & \int_{t}^{T}\left\{\frac{1}{2} \nabla \cdot\left(\sigma \sigma^{T}\left(V_{x_{k}}^{\varepsilon}\right)_{x}\right)+\left\langle\bar{b},\left(V_{x_{k}}^{\varepsilon}\right)_{x}\right\rangle+\bar{b}^{0} V_{x_{k}}^{\varepsilon}+\nabla \cdot\left(\sigma q_{x_{k}}^{\varepsilon}\right)+\left\langle\bar{\sigma}^{0}, q_{x_{k}}^{\varepsilon}\right\rangle\right. \\
& -\psi^{\prime}\left(\frac{V^{\varepsilon}}{\varepsilon}\right) \frac{V_{x_{k}}^{\varepsilon}}{\varepsilon}+\frac{1}{2} \nabla \cdot\left(\left(\sigma \sigma^{T}\right)_{x_{k}} V_{x}^{\varepsilon}\right)+\left\langle\bar{b}_{x_{k}}, V_{x}^{\varepsilon}\right\rangle+\bar{b}_{x_{k}}^{0} V^{\varepsilon} \\
& \left.+\nabla \cdot\left(\sigma_{x_{k}} q^{\varepsilon}\right)+\left\langle\bar{\sigma}_{x_{k}}^{0}, q^{\varepsilon}\right\rangle+\bar{g}_{x_{k}}\right\} d r-\int_{t}^{T}\left\langle q_{x_{k}}^{\varepsilon}, d W(r)\right\rangle .
\end{aligned}
$$

$(5.21)$

We have the following result.

Lemma 5.5. Let (H3) hold and $\left(V^{\varepsilon}, q^{\varepsilon}\right)$ be the classical solution to BSPDE (5.13). Then there exists a constant $C>0$, independent of $\varepsilon>0$ such that

$$
\begin{aligned}
& \mathbb{E} \int_{\mathbb{R}^{n}}\left|V_{x}^{\varepsilon}(t, x)\right|^{2} d x \\
& +\mathbb{E} \int_{t}^{T} \int_{\mathbb{R}^{n}}\left\{\left|\sigma(r, x)^{T} V_{x x}^{\varepsilon}(r, x)+q_{x}^{\varepsilon}(r, x)^{T}\right|^{2}+\frac{\left|V_{x}^{\varepsilon}(r, x)\right|^{2}}{\varepsilon} \psi^{\prime}\left(\frac{V^{\varepsilon}(r, x)}{\varepsilon}\right)\right\} d x d r \\
& \leq C \mathbb{E} \int_{t}^{T} \int_{\mathbb{R}^{n}}\left(|\bar{g}(r, x)|^{2}+\left|\bar{g}_{x}(r, x)\right|^{2}\right) d x d r \quad \forall \varepsilon>0, t \in[0, T] .
\end{aligned}
$$

Note that, in [16] (see [17] also), to obtain an estimate similar to (5.22), a symmetric condition was assumed. Our proof above removes such a condition. Next, the result gives the monotonicity of the sequence $\left\{V^{\varepsilon}(\cdot, \cdot)\right\}_{\varepsilon>0}$ in $\varepsilon>0$.

Lemma 5.6. Let (H3) hold and $0<\varepsilon<\delta$. Let $\left(V^{\varepsilon}, q^{\varepsilon}\right)$ be the adapted classical solution of BSPDE (5.13) and $\left(V^{\delta}, q^{\delta}\right)$ be the adapted classical solution of (5.13) with $\varepsilon$ replaced by $\delta$. Then,

$$
V^{\varepsilon}(t, x) \leq V^{\delta}(t, x), \quad(t, x) \in[0, T] \times \mathbb{R}^{n}, \text { a.s. }
$$

Proof. We observe that, by the definition of $\psi(\cdot)$, one has

$$
\psi\left(\frac{v}{\varepsilon}\right) \geq \psi\left(\frac{v}{\delta}\right), \quad \forall 0<\varepsilon<\delta, \quad v \in \mathbb{R} .
$$

Copyright (c) by SIAM. Unauthorized reproduction of this article is prohibited. 
Hence, by letting

$$
V^{\varepsilon, \delta}=V^{\varepsilon}-V^{\delta}, \quad q^{\varepsilon, \delta}=q^{\varepsilon}-q^{\delta}
$$

we have

$$
\begin{aligned}
V^{\varepsilon, \delta}(t, x)= & \int_{t}^{T}\left\{\frac{1}{2} \nabla \cdot\left(\sigma \sigma^{T} V_{x}^{\varepsilon, \delta}\right)+\left\langle\bar{b}, V_{x}^{\varepsilon, \delta}\right\rangle+\bar{b}^{0} V^{\varepsilon, \delta}+\nabla \cdot\left(\sigma q^{\varepsilon, \delta}\right)+\left\langle\bar{\sigma}^{0}, q^{\varepsilon, \delta}\right\rangle\right. \\
& \left.-\left[\psi\left(\frac{V^{\varepsilon}}{\varepsilon}\right)-\psi\left(\frac{V^{\delta}}{\delta}\right)\right]\right\} d r-\int_{t}^{T}\left\langle q^{\varepsilon, \delta}, d W(r)\right\rangle, \quad(t, x) \in[0, T] \times \mathbb{R}^{n} .
\end{aligned}
$$

Note that

$$
\begin{aligned}
-\left[\psi\left(\frac{V^{\varepsilon}}{\varepsilon}\right)-\psi\left(\frac{V^{\delta}}{\delta}\right)\right]= & -\left[\psi\left(\frac{V^{\varepsilon}}{\varepsilon}\right)-\psi\left(\frac{V^{\delta}}{\varepsilon}\right)+\psi\left(\frac{V^{\delta}}{\varepsilon}\right)-\psi\left(\frac{V^{\delta}}{\delta}\right)\right] \\
= & -\left[\int_{0}^{1} \psi^{\prime}\left(\frac{\lambda V^{\varepsilon}+(1-\lambda) V^{\delta}}{\varepsilon}\right) d \lambda\right] \frac{V^{\varepsilon, \delta}}{\varepsilon} \\
& -\left[\psi\left(\frac{V^{\delta}}{\varepsilon}\right)-\psi\left(\frac{V^{\delta}}{\delta}\right)\right],
\end{aligned}
$$

with

$$
-\left[\psi\left(\frac{V^{\delta}}{\varepsilon}\right)-\psi\left(\frac{V^{\delta}}{\delta}\right)\right] \leq 0
$$

Hence, by a comparison theorem for linear BSPDEs (see [16]), we have

$$
V^{\varepsilon, \delta}(t, x) \leq 0, \quad(t, x) \in[0, T] \times \mathbb{R}^{n} \quad \text { a.s. }
$$

which proves our conclusion.

Having estimates $(5.18)-(5.20),(5.22)$, and $(5.23)$ for $\left(V^{\varepsilon}, q^{\varepsilon}\right)$, we are now ready to prove the following result.

THEOREM 5.7. Let (H3) hold. Then, BSPDVI (4.20) admits an adapted weak solution $(\bar{V}, \bar{q}, \bar{\zeta})$.

Proof. First of all, by Lemma 5.6, we see that

$$
\lim _{\varepsilon \rightarrow 0} V^{\varepsilon}(t, x)=\bar{V}(t, x) \quad \forall(t, x) \in[0, T] \times \mathbb{R}^{n} \text { a.s. }
$$

for some $\mathbb{F}$-adapted random field $\bar{V}(\cdot, \cdot)$, with $(t, x) \mapsto \bar{V}(t, x)$ being upper semicontinuous a.s. Next, by taking the $2 m$ th root in both sides of (5.18) and then sending $m \rightarrow \infty$, we get

$$
\operatorname{esssup}_{(t, x, \omega) \in[0, T] \times \mathbb{R}^{n} \times \Omega}\left|V^{\varepsilon}(t, x, \omega)\right| \leq C \underset{(t, x, \omega) \in[0, T] \times \mathbb{R}^{n} \times \Omega}{\operatorname{esssup}}|\bar{g}(t, x, \omega)| \quad \forall \varepsilon>0 .
$$

Hence, by taking $m=1$ in (5.18) and combining it with (5.22) and (5.31), we have

$$
\begin{aligned}
& \operatorname{lissup}_{(t, x, \omega) \in[0, T] \times \mathbb{R}^{n} \times \Omega}\left|V^{\varepsilon}(t, x, \omega)\right|^{2}+\sup _{t \in[0, T]} \mathbb{E} \int_{\mathbb{R}^{n}}\left|V_{x}^{\varepsilon}(t, x)\right|^{2} d x \\
& +\mathbb{E} \int_{0}^{T} \int_{\mathbb{R}^{n}}\left\{\left|\sigma(r, x)^{T} V_{x x}^{\varepsilon}(r, x)+q_{x}^{\varepsilon}(r, x)^{T}\right|^{2}+\left|q^{\varepsilon}(r, x)\right|^{2}\right. \\
& \left.+\frac{V^{\varepsilon}(r, x)\left[V^{\varepsilon}(r, x)-\varepsilon\right]+\left|V_{x}^{\varepsilon}(r, x)\right|^{2}}{\varepsilon} I_{\left\{V^{\varepsilon} \geq 2 \varepsilon\right\}}\right\} d x d r \\
& \leq C\left[\operatorname{esssup}_{(t, x, \omega) \in[0, T] \times \mathbb{R}^{n} \times \Omega}|\bar{g}(t, x, \omega)|^{2}+\mathbb{E} \int_{0}^{T} \int_{\mathbb{R}^{n}}\left|\bar{g}_{x}(r, x)\right|^{2} d x d r\right] \quad \forall \varepsilon>0 .
\end{aligned}
$$

Copyright $@$ ㅇ by SIAM. Unauthorized reproduction of this article is prohibited. 
Next, by (5.32) and (5.20), together with the above, we know that with the $\bar{V}$ as in (5.30), and for some $\bar{q}$ and $\bar{\zeta}$, one has

$$
\left\{\begin{array}{l}
V^{\varepsilon} \rightarrow \bar{V} \quad \text { strongly in } L_{\mathbb{F}}^{2}\left(0, T ; L^{2}\left(\mathbb{R}^{n}\right)\right) \\
V_{x}^{\varepsilon} \rightarrow \bar{V}_{x} \quad \text { weakly in } L_{\mathbb{F}}^{2}\left(0, T ; L^{2}\left(\mathbb{R}^{n} ; \mathbb{R}^{1 \times n}\right)\right), \\
q^{\varepsilon} \rightarrow \bar{q} \quad \text { weakly in } L_{\mathbb{F}}^{2}\left(0, T ; L^{2}\left(\mathbb{R}^{n} ; \mathbb{R}^{d}\right)\right) \\
\psi\left(\frac{V^{\varepsilon}}{\varepsilon}\right) \rightarrow \bar{\zeta} \quad \text { weakly in } L_{\mathbb{F}}^{2}\left(0, T ; L^{2}\left(\mathbb{R}^{n}\right)\right)
\end{array}\right.
$$

and

$$
\mathbb{E} \int_{0}^{T} \int_{\mathbb{R}^{n}}\left\{\left[V^{\varepsilon}(r, x)-\varepsilon\right]^{2}+\left|V_{x}^{\varepsilon}(r, x)\right|^{2}\right\} I_{\left(V^{\varepsilon} \geq 2 \varepsilon\right)} d x d r \leq C \varepsilon
$$

This yields

$$
\begin{cases}{\left[\left(V^{\varepsilon}-\varepsilon\right)+\left|V_{x}^{\varepsilon}\right|\right] I_{\left\{V^{\varepsilon} \geq 2 \varepsilon\right\}} \rightarrow 0} & \text { strongly in } L_{\mathbb{F}}^{2}\left(0, T ; L^{2}\left(\mathbb{R}^{n}\right)\right) \\ {\left[V^{\varepsilon}(t, x)+\left|V_{x}^{\varepsilon}(t, x)\right|\right] \bar{I}_{\left\{V^{\varepsilon} \geq 2 \varepsilon\right\}} \rightarrow 0} & \text { a.e. }(t, x) \in[0, T] \times \mathbb{R}^{n} \text { a.s. }\end{cases}
$$

Then it is necessary that

$$
\bar{V}(t, x) \leq 0, \quad(t, x) \in[0, T] \times \mathbb{R}^{n}, \quad \text { a.s. },
$$

and together with (5.30), we have

$$
\{\bar{V}<0\}=\bigcup_{\varepsilon>0}\left\{V^{\varepsilon}<0\right\} .
$$

Also, it is necessary that

$$
\bar{\zeta}(t, x) \geq 0, \quad \text { a.e. }(t, x) \in[0, T] \times \mathbb{R}^{n}, \text { a.s. }
$$

On the other hand, by applying the dominated convergence theorem to (5.19), we obtain

$$
\begin{cases}\left(V^{\varepsilon}\right)^{+} \psi\left(\frac{V^{\varepsilon}}{\varepsilon}\right)=V^{\varepsilon} \psi\left(\frac{V^{\varepsilon}}{\varepsilon}\right) \rightarrow 0 & \text { strongly in } L_{\mathbb{F}}^{1}\left(0, T ; L^{1}\left(\mathbb{R}^{n}\right)\right), \\ V^{\varepsilon}(t, x) \psi\left(\frac{V^{\varepsilon}(t, x)}{\varepsilon}\right) \rightarrow 0 & \text { a.e. }(t, x) \in[0, T] \times \mathbb{R}^{n} \text { a.s. }\end{cases}
$$

Hence, it is necessary that

$$
\bar{V}(t, x) \bar{\zeta}(t, x)=0, \quad \text { a.e. }(t, x) \in[0, T] \times \mathbb{R}^{n} \text {, a.s. }
$$

Now, for any $\varphi(\cdot) \in C_{0}^{\infty}\left(\mathbb{R}^{n}\right)$, we have from (5.13) that

$$
\begin{aligned}
\int_{\mathbb{R}^{n}} V^{\varepsilon}(t, x) \varphi(x) d x= & \int_{t}^{T} \int_{\mathbb{R}^{n}}\left\{-\left\langle\frac{1}{2} \sigma^{T} V_{x}^{\varepsilon}+q^{\varepsilon}, \sigma^{T} \varphi_{x}\right\rangle+\left[\left\langle\bar{b}, V_{x}^{\varepsilon}\right\rangle+\bar{b}^{0} V^{\varepsilon}\right.\right. \\
& \left.\left.+\left\langle\bar{\sigma}^{0}, q^{\varepsilon}\right\rangle+\bar{g}-\psi\left(\frac{V^{\varepsilon}}{\varepsilon}\right)\right] \varphi\right\} d x d r \\
& -\int_{t}^{T}\left\langle\int_{\mathbb{R}^{n}} q^{\varepsilon} \varphi d x, d W(r)\right\rangle, \quad t \in[0, T] .
\end{aligned}
$$

Copyright $@$ by SIAM. Unauthorized reproduction of this article is prohibited. 
Then, letting $\varepsilon \rightarrow 0$, along a sequence, we obtain

$$
\begin{aligned}
\int_{\mathbb{R}^{n}} \bar{V}(t, x) \varphi(x) d x= & \int_{t}^{T} \int_{\mathbb{R}^{n}}\left\{-\left\langle\frac{1}{2} \sigma^{T} \bar{V}_{x}+\bar{q}, \sigma^{T} \varphi_{x}\right\rangle\right. \\
& \left.+\left[\left\langle\bar{b}, \bar{V}_{x}\right\rangle+\bar{b}^{0} \bar{V}+\left\langle\bar{\sigma}^{0}, \bar{q}\right\rangle+\bar{g}-\bar{\zeta}\right] \varphi\right\} d x d r \\
& -\int_{t}^{T}\left\langle\int_{\mathbb{R}^{n}} \bar{q} \varphi d x, d W(r)\right\rangle, \quad t \in[0, T] .
\end{aligned}
$$

Hence, $(\bar{V}, \bar{q}, \bar{\zeta})$ is the adapted weak solution of $(4.20)$.

From the above, we see that, for any $\varphi(\cdot) \in C_{0}^{\infty}\left(\mathbb{R}^{n}\right)$, map $t \mapsto \int_{\mathbb{R}^{n}} \bar{V}(t, x) \varphi(x) d x$ is continuous.

6. Identification of the value function. In this section, we are going to identify the weak adapted solution of BSPDVI as the value function $V(\cdot, \cdot)$ of Problem (S). Suppose $\left(V^{\varepsilon}(\cdot), q^{\varepsilon}(\cdot)\right)$ is the adapted classical solution of (5.13). Let

$$
\left\{\begin{array}{l}
\widetilde{V}^{\varepsilon}(t, x)=\left(1+|x|^{p}\right) V^{\varepsilon}(t, x)+h(t, x), \\
\widetilde{q}^{\varepsilon}(t, x)=\left(1+|x|^{p}\right) q^{\varepsilon}(t, x)+\mu(t, x),
\end{array} \quad(t, x) \in[0, T] \times \mathbb{R}^{n} .\right.
$$

Then

$$
\begin{aligned}
\widetilde{V}^{\varepsilon}(t, x)= & h(T, x)+\int_{t}^{T}\left\{\frac{1}{2} \operatorname{tr}\left[\sigma(r, x) \sigma(r, x)^{T} \widetilde{V}_{x x}^{\varepsilon}(r, x)\right]+\left\langle b(r, x), \widetilde{V}_{x}^{\varepsilon}(r, x)\right\rangle\right. \\
& \left.+\operatorname{tr}\left[\sigma(r, x) \widetilde{q}_{x}^{\varepsilon}(r, x)\right]-\psi\left(\frac{\widetilde{V}^{\varepsilon}(r, x)-h(r, x)}{\varepsilon}\right)+g(r, x)\right\} d r \\
& -\int_{t}^{T}\left\langle\widetilde{q}^{\varepsilon}(r, x), d W(r)\right\rangle \\
\equiv & h(T, x)+\int_{t}^{T} \widetilde{q}^{0, \varepsilon}(r, x) d r-\int_{t}^{T}\left\langle\widetilde{q}^{\varepsilon}(r, x), d W(r)\right\rangle, \quad(t, x) \in[s, T] \times \mathbb{R}^{n} .
\end{aligned}
$$

Consequently, by Itô-Kunita's formula, we have

$$
\begin{aligned}
\widetilde{V}^{\varepsilon}(t, X(t ; s, \xi))= & \widetilde{V}^{\varepsilon}(s, \xi)+\int_{s}^{t}\left\{\widetilde{q}^{0, \varepsilon}(r, X(r ; s, \xi))\right. \\
& +\left\langle b(r, X(r ; s, \xi)), \widetilde{V}_{x}^{\varepsilon}(r, X(r ; s, \xi))\right\rangle \\
& +\frac{1}{2} \operatorname{tr}\left[\sigma(r, X(r ; s, \xi)) \sigma(r, X(r ; s, \xi))^{T} \widetilde{V}_{x x}^{\varepsilon}(r, X(r ; s, \xi))\right] \\
& \left.+\operatorname{tr}\left[\sigma(r, X(r ; s, \xi)) \widetilde{q}_{x}^{\varepsilon}(r, X(r ; s, \xi))\right]\right\} d r \\
& +\int_{s}^{t}\left\langle\widetilde{q}^{\varepsilon}(r, X(r ; s, \xi))+\sigma(r, X(r ; s, \xi))^{T} \widetilde{V}_{x}^{\varepsilon}(r, X(r ; s, \xi)), d W(r)\right\rangle \\
= & \widetilde{V}^{\varepsilon}(s, \xi)+\int_{s}^{t}\left\{\psi\left(\frac{\widetilde{V}^{\varepsilon}(r, X(r ; s, \xi))-h(r, X(r ; s, \xi))}{\varepsilon}\right)\right. \\
& +\int_{s}^{t}\left\langle\widetilde{q}^{\varepsilon}(r, X(r ; s, \xi))+\sigma(r, X(r ; s, \xi))^{T} \widetilde{V}_{x}^{\varepsilon}(r, X(r ; s, \xi)), d W(r)\right\rangle .
\end{aligned}
$$

Copyright $@$ by SIAM. Unauthorized reproduction of this article is prohibited. 
Thus, for any $\tau \in \mathcal{S}[s, T]$,

$$
\begin{aligned}
J_{s, \xi}(\tau)= & \mathbb{E}\left[\int_{s}^{\tau} g(r, X(r ; s, \xi)) d r+h(\tau, X(\tau ; s, \xi)) \mid \mathcal{F}_{s}\right] \\
= & \widetilde{V}^{\varepsilon}(s, \xi)+\mathbb{E}\left[\int_{s}^{\tau} \psi\left(\frac{\widetilde{V}^{\varepsilon}(r, X(r ; s, \xi))-h(r, X(r ; s, \xi))}{\varepsilon}\right) d r \mid \mathcal{F}_{s}\right] \\
& +\mathbb{E}\left[h(\tau, X(\tau ; s, \xi))-\widetilde{V}^{\varepsilon}(\tau, X(\tau, s, \xi)) \mid \mathcal{F}_{s}\right] .
\end{aligned}
$$

By our discussion above, we know that under (H3),

$$
\widetilde{V}^{\varepsilon}(t, x) \downarrow V^{*}(t, x), \quad \forall(t, x) \in[0, T] \times \mathbb{R}^{n}, \text { a.s. },
$$

with $(t, x) \mapsto V^{*}(t, x)$ being upper semicontinuous, and by (5.36),

$$
V^{*}(s, \xi)=\left(1+|x|^{p}\right) \bar{V}(s, \xi)+h(s, \xi) \leq h(s, \xi), \quad \text { a.s., } \forall(s, \xi) \in \mathcal{D}^{p} .
$$

Now, sending $\varepsilon \rightarrow 0$ in (6.4), we have

$$
\begin{aligned}
J_{s, \xi}(\tau)= & V^{*}(s, \xi)+\mathbb{E}\left[\int_{s}^{\tau} \zeta^{*}(r, X(r ; s, \xi)) d r \mid \mathcal{F}_{s}\right] \\
& +\mathbb{E}\left[h(\tau, X(\tau ; s, \xi))-V^{*}(\tau, X(\tau, s, \xi)) \mid \mathcal{F}_{s}\right]
\end{aligned}
$$

with

$$
\zeta^{*}(t, x) \in \beta\left(V^{*}(t, x)-h(t, x)\right), \quad(t, x) \in[0, T] \times \mathbb{R}^{n}, \text { a.s. }
$$

Hence,

$$
J_{s, \xi}(\tau) \geq V^{*}(s, \xi), \quad(s, \xi) \in \mathcal{D}^{p}, \quad \tau \in \mathcal{S}[s, T]
$$

which leads to

$$
V(s, \xi) \geq V^{*}(s, \xi), \quad(s, \xi) \in \mathcal{D}^{p} .
$$

Next, let $(s, \xi) \in \mathcal{D}^{p}$ be fixed. We define

$$
\tau^{*}(s, \xi)=\inf \left\{r \in[s, T] \mid V^{*}(r, X(r ; s, \xi))=h(r, X(r ; s, \xi))\right\} .
$$

Since $t \mapsto V^{*}(r, X(r ; s, \xi))-h(r, X(r ; s, \xi))$ is $\mathbb{F}$-progressively measurable, by Début Theorem (see [6], [19]), $\tau^{*}(s, \xi) \in \mathcal{S}[s, T]$. Then, taking $\tau=\tau^{*}(s, \xi)$ in (6.7), we obtain

$$
V(s, \xi) \leq J_{s, \xi}\left(\tau^{*}(s, \xi)\right)=V^{*}(s, \xi) .
$$

Hence, combining the above with (6.10), one must have the following:

$$
V^{*}(s, \xi)=V(s, \xi) \quad \forall(s, \xi) \in \mathcal{D}^{p} .
$$

That means $V^{*}(\cdot, \cdot)$ must be the value function $V(\cdot, \cdot)$ of Problem (S). Consequently, $(t, x) \mapsto V^{*}(t, x)=V(t, x)$ must be continuous itself. Moreover, the smallest optimal stopping time can be identified through (3.4).

Copyright (c) by SIAM. Unauthorized reproduction of this article is prohibited. 
7. Appendix. In this appendix, we collect some proofs.

Sketch proof of Theorem 3.1. (i) For any $\tau \in \mathcal{S}[s, T]$, we have

$$
V(s, \xi) \leq J_{s, \xi}(\tau) \equiv \mathbb{E}\left[\int_{s}^{\tau} g(r, X(r ; s, \xi)) d r+h(\tau, X(\tau)) \mid \mathcal{F}_{s}\right] .
$$

In particular, taking $\tau=s$, one obtains (3.1). Next, for any $\tau \in \mathcal{S}[s, T]$, take $\theta \in$ $\mathcal{S}[\tau, T]$. One has

$$
V(s, \xi) \leq J_{s, \xi}(\theta)=\mathbb{E}\left[\int_{s}^{\tau} g(r, X(r ; s, \xi)) d t+J_{\tau, X(\tau ; s, \xi)}(\theta) \mid \mathcal{F}_{s}\right] .
$$

Taking infimum with respect to $\theta \in \mathcal{S}[\tau, T]$ yields

$$
V(s, \xi) \leq \mathbb{E}\left[\int_{s}^{\tau} g(r, X(r ; s, \xi)) d r+V(\tau, X(\tau ; s, \xi)) \mid \mathcal{F}_{s}\right] .
$$

Hence, (3.2) holds.

(ii) Suppose $\bar{\theta} \in \mathcal{S}[\theta, T]$ is optimal for initial point $(s, \xi) \in \mathcal{D}^{p}$. Then

$$
\begin{aligned}
V(s, \xi) & =J_{s, \xi}(\bar{\theta}) \geq \mathbb{E}\left[\int_{s}^{\bar{\theta}} g(r, X(r ; s, \xi)) d r+V(\bar{\theta}, X(\bar{\theta} ; s, \xi)) \mid \mathcal{F}_{s}\right] \\
& \geq \inf _{\tau \in \mathcal{S}[s, T]} \mathbb{E}\left[\int_{s}^{\tau} g(r, X(r ; s, \xi)) d r+V(\tau, X(\tau ; s, \xi)) \mid \mathcal{F}_{s}\right] \geq V(s, \xi) .
\end{aligned}
$$

Hence, the equalities in the above have to hold, which implies

$$
\mathbb{E}\left[V(\bar{\theta}, X(\bar{\theta} ; s, \xi)) \mid \mathcal{F}_{s}\right]=\mathbb{E}\left[h(\bar{\theta}, X(\bar{\theta} ; s, \xi)) \mid \mathcal{F}_{s}\right] .
$$

Combining the fact

$$
V(\bar{\theta}, X(\bar{\theta} ; s, \xi)) \leq h(\bar{\theta}, X(\bar{\theta} ; s, \xi)) \quad \text { a.s. }
$$

we obtain (3.3). Next, for (3.5), if there exists a $\Omega_{0} \subseteq\{V(s, \xi)<h(s, \xi)\}$, with $\mathbb{P}\left(\Omega_{0}\right)>0$ such that

$$
\bar{\tau}(s, \xi)=s \quad \text { on } \Omega_{0},
$$

then, trivially,

$$
V(s, \xi(\omega))=h(s, \xi(\omega)), \quad \omega \in \Omega_{0},
$$

which contradicts the choice of $\Omega_{0}$. Conversely, if $\Omega_{0} \subseteq\{\bar{\tau}(s, \xi)>s\}$, with $\mathbb{P}\left(\Omega_{0}\right)>0$ such that (7.7) holds, then (7.6) has to be true (by definition of $\bar{\tau}(s, \xi)$ ), a contradiction to the choice of $\Omega_{0}$. Hence, (3.5) holds.

We now show (3.6). To this end, let $(s, \xi) \in \mathcal{D}^{p}$. Define $\bar{\tau}(s, \xi)$ by $(3.4)$, and suppose $\mathbb{P}\{s<\bar{\tau}(s, \xi)\}>0$. The case $\theta=\bar{\tau}(s, \xi)$ is trivial. Thus, we fix $\theta \in$ $\mathcal{S}[s, \bar{\tau}(s, \xi)$ ), and let $\tau \in \mathcal{S}[\theta, \bar{\tau}(s, \xi)]$. From (3.3), we know that any $\mu \in \mathcal{S}[\theta, T]$, with $\mathbb{P}\{\mu<\tau\}>0$ is not optimal for initial point $(\theta, X(\theta ; s, \xi))$. Hence,

$$
\begin{aligned}
V(\theta, X(\theta ; s, \xi)) & =\inf _{\mu \in \mathcal{S}[\tau, T]} \mathbb{E}\left[\int_{\theta}^{\tau} g(r, X(r ; s, \xi)) d r+J_{\tau, X(\tau ; s, \xi)}(\mu) \mid \mathcal{F}_{\theta}\right] \\
& =\mathbb{E}\left[\int_{\theta}^{\tau} g(r, X(r ; s, \xi)) d r+V(\tau, X(\tau ; s, \xi)) \mid \mathcal{F}_{\theta}\right],
\end{aligned}
$$

proving (3.6).

Copyright $@$ by SIAM. Unauthorized reproduction of this article is prohibited. 
Finally, by taking $\theta=s$ and $\tau=\bar{\tau}(s, \xi)$ in (3.6), we see that

$V(s, \xi)=\mathbb{E}\left[\int_{s}^{\bar{\tau}(s, x)} g(s, X(s ; s, \xi)) d s+h(\bar{\tau}(s, \xi), X(\bar{\tau}(s, x) ; s, \xi)) \mid \mathcal{F}_{s}\right]=J_{s, \xi}(\bar{\tau}(s, \xi))$,

which means that $\bar{\tau}(s, \xi)$ is an optimal stopping time of Problem (S) for the initial point $(s, \xi)$, and it must be the smallest one.

Proof of Lemma 5.3. By (5.16), we may assume that

$$
\frac{\theta^{\prime}(\rho)}{\theta^{\prime \prime}(\rho)}=\frac{\theta^{\prime}(\rho)}{\theta^{\prime \prime}(\rho)} I_{\left\{\theta^{\prime \prime}(\rho)>0\right\}} \quad \forall \rho \in \mathbb{R}
$$

since $\theta: \mathbb{R} \rightarrow[0, \infty)$ is convex and piecewise smooth. Applying Itô's formula to $\theta\left(V^{\varepsilon}\right)$, we have

$$
\begin{aligned}
&-\mathbb{E} \int_{\mathbb{R}^{n}} \theta\left(V^{\varepsilon}(t, x)\right) d x \\
&=- \mathbb{E} \int_{t}^{T} \int_{\mathbb{R}^{n}}\left\{\theta ^ { \prime } ( V ^ { \varepsilon } ) \left[\frac{1}{2} \nabla \cdot\left(\sigma \sigma^{T} V_{x}^{\varepsilon}\right)+\left\langle\bar{b}, V_{x}^{\varepsilon}\right\rangle+\bar{b}^{0} V^{\varepsilon}+\nabla \cdot\left(\sigma q^{\varepsilon}\right)+\left\langle\bar{\sigma}^{0}, q^{\varepsilon}\right\rangle\right.\right. \\
&\left.\left.+\bar{g}-\psi\left(\frac{V^{\varepsilon}}{\varepsilon}\right)\right]-\frac{1}{2} \theta^{\prime \prime}\left(V^{\varepsilon}\right)\left|q^{\varepsilon}\right|^{2}\right\} d x d r \\
&= \frac{1}{2} \mathbb{E} \int_{t}^{T} \int_{\mathbb{R}^{n}}\left\{\theta^{\prime \prime}\left(V^{\varepsilon}\right)\left|\sigma^{T} V_{x}^{\varepsilon}\right|^{2}+2\left\langle q^{\varepsilon}, \theta^{\prime \prime}\left(V^{\varepsilon}\right) \sigma^{T} V_{x}^{\varepsilon}-\theta^{\prime}\left(V^{\varepsilon}\right) \bar{\sigma}^{0}\right\rangle+\theta^{\prime \prime}\left(V^{\varepsilon}\right)\left|q^{\varepsilon}\right|^{2}\right. \\
&\left.+2(\nabla \cdot \bar{b}) \theta\left(V^{\varepsilon}\right)+2 \theta^{\prime}\left(V^{\varepsilon}\right)\left[\psi\left(\frac{V^{\varepsilon}}{\varepsilon}\right)-\bar{b}^{0} V^{\varepsilon}-\bar{g}\right]\right\} d x d r \\
&= \frac{1}{2} \mathbb{E} \int_{t}^{T} \int_{\mathbb{R}^{n}}\left\{\theta^{\prime \prime}\left(V^{\varepsilon}\right)\left[\left|\sigma^{T} V_{x}^{\varepsilon}\right|^{2}+2\left\langle q^{\varepsilon}, \sigma^{T} V_{x}^{\varepsilon}-\frac{\theta^{\prime}\left(V^{\varepsilon}\right)}{\theta^{\prime \prime}\left(V^{\varepsilon}\right)} \bar{\sigma}^{0}\right\rangle+\left|q^{\varepsilon}\right|^{2}\right]\right. \\
&\left.+2(\nabla \cdot \bar{b}) \theta\left(V^{\varepsilon}\right)+2 \theta^{\prime}\left(V^{\varepsilon}\right)\left[\psi\left(\frac{V^{\varepsilon}}{\varepsilon}\right)-\bar{b}^{0} V^{\varepsilon}-\bar{g}\right]\right\} d x d r \\
&= \frac{1}{2} \mathbb{E} \int_{t}^{T} \int_{\mathbb{R}^{n}}\left\{\theta^{\prime \prime}\left(V^{\varepsilon}\right)\left|\sigma^{T} V_{x}^{\varepsilon}+q^{\varepsilon}-\frac{\theta^{\prime}\left(V^{\varepsilon}\right)}{\theta^{\prime \prime}\left(V^{\varepsilon}\right)} \bar{\sigma}^{0}\right|^{2}+2\left[(\nabla \cdot \bar{b})-\nabla \cdot\left(\sigma \bar{\sigma}^{0}\right)\right] \theta\left(V^{\varepsilon}\right)\right. \\
&\left.-\frac{\left|\theta^{\prime}\left(V^{\varepsilon}\right)\right|^{2}}{\theta^{\prime \prime}\left(V^{\varepsilon}\right)}\left|\bar{\sigma}^{0}\right|^{2}+\theta^{\prime}\left(V^{\varepsilon}\right)\left[\psi\left(\frac{V^{\varepsilon}}{\varepsilon}\right)-\bar{b}^{0} V^{\varepsilon}-\bar{g}\right]\right\} d x d r \\
& \geq \frac{1}{2} \mathbb{E} \int_{t}^{T} \int_{\mathbb{R}^{n}}\left\{\frac{\theta^{\prime \prime}\left(V^{\varepsilon}\right)}{2}\left|\sigma^{T} V_{x}^{\varepsilon}+q^{\varepsilon}\right|^{2}+2\left[(\nabla \cdot \bar{b})-\nabla \cdot\left(\sigma \bar{\sigma}^{0}\right)\right] \theta\left(V^{\varepsilon}\right)\right. \\
&\left.-\frac{2\left|\theta^{\prime}\left(V^{\varepsilon}\right)\right|^{2}}{\theta^{\prime \prime}\left(V^{\varepsilon}\right)}\left|\bar{\sigma}^{0}\right|^{2}+\theta^{\prime}\left(V^{\varepsilon}\right)\left[\psi\left(\frac{V^{\varepsilon}}{\varepsilon}\right)-\bar{b}^{0} V^{\varepsilon}-\bar{g}\right]\right\} d x d r \\
& \geq \frac{1}{2} \mathbb{E} \int_{t}^{T} \int_{\mathbb{R}^{n}}\left\{\theta^{\prime \prime}\left(V^{\varepsilon}\right)\left|\sigma^{T} V_{x}^{\varepsilon}+q^{\varepsilon}\right|^{2}+\theta^{\prime}\left(V^{\varepsilon}\right) \psi\left(\frac{V^{\varepsilon}}{\varepsilon}\right)\right. \\
&\left.-C \theta\left(V^{\varepsilon}\right)-C\left|\theta^{\prime}\left(V^{\varepsilon}\right)\right|\left(\left|V^{\varepsilon}\right|+|\bar{g}|\right)\right\} d x d r . \\
&(7111)
\end{aligned}
$$

In the above, we have used the fact that $|a-b| \geq \frac{1}{2}|a|-|b|$. Note that, under our conditions,

$$
\theta^{\prime}(\rho) \psi\left(\frac{\rho}{\varepsilon}\right) \geq 0 \quad \text { a.e. } \rho \in \mathbb{R} .
$$

Hence, by Gronwall's inequality, we obtain (5.17).

Copyright (c) by SIAM. Unauthorized reproduction of this article is prohibited. 
Proof of Lemma 5.4. For any $m \geq 1$, taking

$$
\theta(\rho)=|\rho|^{2 m}, \quad \rho \in \mathbb{R}^{n} .
$$

Then (5.15) and (5.16) hold. Hence, by Lemma 5.3, we obtain

$$
\begin{gathered}
\mathbb{E} \int_{\mathbb{R}^{n}} V^{\varepsilon}(t, x)^{2 m} d x+\mathbb{E} \int_{t}^{T} \int_{\mathbb{R}^{n}}\left\{2 m(2 m-1)\left(V^{\varepsilon}\right)^{2 m-2}\left|\sigma^{T} V_{x}^{\varepsilon}+q^{\varepsilon}\right|^{2}\right. \\
\left.(7.14)+2 m\left(V^{\varepsilon}\right)^{2 m-1} \psi\left(\frac{V^{\varepsilon}}{\varepsilon}\right)\right\} d x d r \leq C \mathbb{E} \int_{t}^{T} \int_{\mathbb{R}^{n}} 2 m\left|V^{\varepsilon}\right|^{2 m-1} \mid\left(\left|V^{\varepsilon}\right|+|\bar{g}|\right) d x d r \\
\leq C \mathbb{E} \int_{t}^{T} \int_{\mathbb{R}^{n}}\left\{(4 m-1)\left(V^{\varepsilon}\right)^{2 m}+|\bar{g}|^{2 m}\right\} d x d r .
\end{gathered}
$$

Then, by Gronwall's inequality, one has

$$
\begin{array}{r}
\mathbb{E} \int_{\mathbb{R}^{n}} V^{\varepsilon}(t, x)^{2 m} d x+\mathbb{E} \int_{t}^{T} \int_{\mathbb{R}^{n}}\left\{2 m(2 m-1)\left(V^{\varepsilon}\right)^{2 m-2}\left|\sigma^{T} V_{x}^{\varepsilon}+q^{\varepsilon}\right|^{2}\right. \\
\left.+2 m\left(V^{\varepsilon}\right)^{2 m-1} \psi\left(\frac{V^{\varepsilon}}{\varepsilon}\right)\right\} d x d r \leq C e^{C m} \mathbb{E} \int_{t}^{T} \int_{\mathbb{R}^{n}} \bar{g}^{2 m} d x d r
\end{array}
$$

which implies (5.18).

Next, by taking

$$
\theta(\rho)=\left(\rho^{+}\right)^{2}, \quad \rho \in \mathbb{R},
$$

we have

$$
\theta^{\prime}(\rho)=2 \rho^{+}, \quad \theta^{\prime \prime}(\rho)=2 I_{\{\rho>0\}},
$$

which leads to

$$
\theta^{\prime}(\rho)^{2}=4\left(\rho^{+}\right)^{2}=2 \theta(\rho) \theta^{\prime \prime}(\rho) .
$$

Thus, (5.15) and (5.16) hold. Hence, by Lemma 5.3, we have

$$
\begin{aligned}
& \mathbb{E} \int_{\mathbb{R}^{n}}\left(V^{\varepsilon}(t, x)^{+}\right)^{2} d x+\mathbb{E} \int_{t}^{T} \int_{\mathbb{R}^{n}}\left\{2 I_{\left\{V^{\varepsilon}>0\right\}}\left|\sigma^{T} V_{x}^{\varepsilon}+q^{\varepsilon}\right|^{2}+2\left(V^{\varepsilon}\right)^{+} \psi\left(\frac{V^{\varepsilon}}{\varepsilon}\right)\right\} d x d r \\
& \leq C \mathbb{E} \int_{t}^{T} \int_{\mathbb{R}^{n}} 2\left(V^{\varepsilon}\right)^{+} \mid\left[\left|V^{\varepsilon}\right|+|\bar{g}|\right] d x d r \leq C \mathbb{E} \int_{t}^{T} \int_{\mathbb{R}^{n}}\left[\left(\left(V^{\varepsilon}\right)^{+}\right)^{2}+|\bar{g}|^{2} I_{\left\{V^{\varepsilon}>0\right\}}\right] d x d r .
\end{aligned}
$$

It follows from Gronwall's inequality that

$$
\begin{gathered}
\mathbb{E} \int_{\mathbb{R}^{n}}\left(V^{\varepsilon}(t, x)^{+}\right)^{2} d x+\mathbb{E} \int_{0}^{T} \int_{\mathbb{R}^{n}}\left\{\left|\sigma^{T} V_{x}^{\varepsilon}+q^{\varepsilon}\right|^{2} I_{\left\{V^{\varepsilon}>0\right\}}+\left(V^{\varepsilon}\right)^{+} \psi\left(\frac{V^{\varepsilon}}{\varepsilon}\right)\right\} d x d r \\
\leq C \mathbb{E} \int_{0}^{T} \int_{\mathbb{R}^{n}}|\bar{g}(r, x)|^{2} I_{\left\{V^{\varepsilon}>0\right\}} d x d r, \quad \forall \varepsilon>0,
\end{gathered}
$$

with $C>0$ independent of $\varepsilon>0$, which leads to (5.19).

Copyright $\odot$ by SIAM. Unauthorized reproduction of this article is prohibited. 
Finally, we take

$$
\theta(\rho)=\rho \psi\left(\frac{\rho}{\varepsilon}\right)=\frac{\rho^{2}}{\varepsilon} \int_{0}^{\rho / \varepsilon} \eta(r) d r-\rho \int_{0}^{\rho / \varepsilon} r \eta(r) d r .
$$

Then

$$
\begin{aligned}
0 & \leq \theta^{\prime}(\rho)=\psi\left(\frac{\rho}{\varepsilon}\right)+\frac{\rho}{\varepsilon} \psi^{\prime}\left(\frac{\rho}{\varepsilon}\right) \\
& =\frac{\rho}{\varepsilon} \int_{0}^{\rho / \varepsilon} \eta(r) d r-\int_{0}^{\rho / \varepsilon} r \eta(r) d r+\frac{\rho}{\varepsilon} \int_{0}^{\rho / \varepsilon} \eta(r) d r \\
& =\frac{2 \rho}{\varepsilon} \int_{0}^{\rho / \varepsilon} \eta(r) d r-\int_{0}^{\rho / \varepsilon} r \eta(r) d r,
\end{aligned}
$$

and

$$
0 \leq \theta^{\prime \prime}(\rho)=\frac{2}{\varepsilon} \psi^{\prime}\left(\frac{\rho}{\varepsilon}\right)+\frac{\rho}{\varepsilon^{2}} \psi^{\prime \prime}\left(\frac{\rho}{\varepsilon}\right)=\frac{2}{\varepsilon} \int_{0}^{\rho / \varepsilon} \eta(r) d r+\frac{\rho}{\varepsilon^{2}} \eta\left(\frac{\rho}{\varepsilon}\right) .
$$

Note that

$$
\theta^{\prime}(\rho)^{2}-C \theta(\rho) \theta^{\prime \prime}(\rho)=\left[\psi\left(\frac{\rho}{\varepsilon}\right)+\frac{\rho}{\varepsilon} \psi^{\prime}\left(\frac{\rho}{\varepsilon}\right)\right]^{2}-C \psi\left(\frac{\rho}{\varepsilon}\right)\left[\frac{2 \rho}{\varepsilon} \psi^{\prime}\left(\frac{\rho}{\varepsilon}\right)+\frac{\rho^{2}}{\varepsilon^{2}} \psi^{\prime \prime}\left(\frac{\rho}{\varepsilon}\right)\right] .
$$

Now, for $\rho \in(-\infty, 0]$, we have

$$
\theta^{\prime}(\rho)^{2}-C \theta(\rho) \theta^{\prime \prime}(\rho)=0
$$

for any $C>0$. For $\rho \in(0, \varepsilon]$,

$$
\theta^{\prime}(\rho)^{2}-C \theta(\rho) \theta^{\prime \prime}(\rho)=\left[\frac{\rho^{3}}{6 \varepsilon^{3}}+\frac{\rho}{\varepsilon} \frac{\rho^{2}}{2 \varepsilon^{2}}\right]^{2}-C \frac{\rho^{3}}{6 \varepsilon^{3}}\left[\frac{2 \rho}{\varepsilon} \frac{\rho^{2}}{2 \varepsilon^{2}}+\frac{\rho^{3}}{\varepsilon^{3}}\right]=\frac{\rho^{6}}{9 \varepsilon^{6}}(4-3 C) \leq 0,
$$

provided $C \geq \frac{4}{3}$. For $\rho \in(\varepsilon, 2 \varepsilon]$, since both $\psi(\cdot)$ and $\psi^{\prime}(\cdot)$ are nondecreasing,

$$
\begin{aligned}
\theta^{\prime}(\rho)^{2}-C \theta(\rho) \theta^{\prime \prime}(\rho) & =\left[\psi\left(\frac{\rho}{\varepsilon}\right)+\frac{\rho}{\varepsilon} \psi^{\prime}\left(\frac{\rho}{\varepsilon}\right)\right]^{2}-C \psi\left(\frac{\rho}{\varepsilon}\right)\left[\frac{2 \rho}{\varepsilon} \psi^{\prime}\left(\frac{\rho}{\varepsilon}\right)+\frac{\rho^{2}}{\varepsilon^{2}} \psi^{\prime \prime}\left(\frac{\rho}{\varepsilon}\right)\right] \\
& \leq\left[\psi(2)+2 \psi^{\prime}(2)\right]^{2}-2 C \psi(1) \psi^{\prime}(1)=9-\frac{C}{6} \leq 0,
\end{aligned}
$$

provided $C \geq 54$, and for $\rho \in[2 \varepsilon, \infty)$,

$$
\begin{aligned}
\theta^{\prime}(\rho)^{2}-C \theta(\rho) \theta^{\prime \prime}(\rho) & =\left[\frac{2 \rho}{\varepsilon}-1\right]^{2}-C\left(\frac{\rho}{\varepsilon}-1\right) \frac{2 \rho}{\varepsilon} \leq \frac{4 \rho^{2}}{\varepsilon^{2}}-\frac{4 \rho}{\varepsilon}+1-2 C \frac{\rho^{2}}{\varepsilon^{2}}+2 C \frac{\rho}{\varepsilon} \\
& =-(C-2) \frac{2 \rho}{\varepsilon}\left(\frac{\rho}{\varepsilon}-1\right)+1 \leq-4(C-2)+1=-4 C+9 \leq 0,
\end{aligned}
$$

provided $C \geq \frac{9}{4}$. Further, we claim that

$$
\frac{\rho}{\varepsilon} \psi^{\prime}\left(\frac{\rho}{\varepsilon}\right) \leq 3 \psi\left(\frac{\rho}{\varepsilon}\right)+1, \quad \rho \in \mathbb{R}
$$

Copyright $@$ ( ) by SIAM. Unauthorized reproduction of this article is prohibited. 
In fact, the above holds for $\rho \leq 0$. Now, for $\rho \in(0, \varepsilon]$,

$$
\frac{\rho}{\varepsilon} \psi^{\prime}\left(\frac{\rho}{\varepsilon}\right)=\frac{\rho^{3}}{2 \varepsilon^{3}}=3 \psi\left(\frac{\rho}{\varepsilon}\right),
$$

and for $\rho \in(\varepsilon, \infty)$,

$$
\frac{\rho}{\varepsilon} \psi^{\prime}\left(\frac{\rho}{\varepsilon}\right)=\frac{\rho}{\varepsilon}\left[1-\frac{(2-\rho)^{2}}{2} I_{\{\rho \leq 2 \varepsilon\}}\right] \leq \frac{\rho}{\varepsilon}=\psi\left(\frac{\rho}{\varepsilon}\right)+1 .
$$

Hence, by Lemma 5.3,

$$
\begin{aligned}
& \mathbb{E} \int_{\mathbb{R}^{n}} V^{\varepsilon}(t, x) \psi\left(\frac{V^{\varepsilon}(t, x)}{\varepsilon}\right) d x+\mathbb{E} \int_{t}^{T} \int_{\mathbb{R}^{n}} \\
&\left\{\left[\frac{2}{\varepsilon} \psi^{\prime}\left(\frac{V^{\varepsilon}}{\varepsilon}\right)+\frac{V^{\varepsilon}}{\varepsilon^{2}} \psi\left(\frac{V^{\varepsilon}}{\varepsilon}\right)\right]\left|\sigma^{T} V_{x}^{\varepsilon}+q^{\varepsilon}\right|^{2}\right. \\
&\left.+\left[\psi\left(\frac{V^{\varepsilon}}{\varepsilon}\right)+\frac{V^{\varepsilon}}{\varepsilon} \psi^{\prime}\left(\frac{V^{\varepsilon}}{\varepsilon}\right)\right] \psi\left(\frac{V^{\varepsilon}}{\varepsilon}\right)\right\} d x d r \\
& \leq C \mathbb{E} \int_{t}^{T} \int_{\mathbb{R}^{n}}\left[\psi\left(\frac{V^{\varepsilon}}{\varepsilon}\right)+\frac{V^{\varepsilon}}{\varepsilon} \psi^{\prime}\left(\frac{V^{\varepsilon}}{\varepsilon}\right)\right]\left(\left|V^{\varepsilon}\right|+|\bar{g}|\right) d x d r \\
& \leq C \mathbb{E} \int_{t}^{T} \int_{\mathbb{R}^{n}} \psi\left(\frac{V^{\varepsilon}}{\varepsilon}\right)\left(V^{\varepsilon}+|\bar{g}|\right) d x d r, \quad t \in[0, T] .
\end{aligned}
$$

Then, by Gronwall's inequality, together with Cauchy-Schwarz inequality, we obtain

$$
\begin{array}{r}
\mathbb{E} \int_{\mathbb{R}^{n}} V^{\varepsilon}(t, x) \psi\left(\frac{V^{\varepsilon}(t, x)}{\varepsilon}\right) d x+\mathbb{E} \int_{t}^{T} \int_{\mathbb{R}^{n}} \psi\left(\frac{V^{\varepsilon}}{\varepsilon}\right)^{2} d x d r \\
\leq C \mathbb{E} \int_{t}^{T} \int_{\mathbb{R}^{n}}|\bar{g}|^{2} d x d r, \quad t \in[0, T] .
\end{array}
$$

This leads to (5.20).

Proof of Lemma 5.5. Applying Itô's formula to $\left|V_{x_{k}}^{\varepsilon}(t, x)\right|^{2}$ yields

$$
\begin{aligned}
& -\mathbb{E} \int_{\mathbb{R}^{n}}\left|V_{x_{k}}^{\varepsilon}(t, x)\right|^{2} d x \\
= & -\mathbb{E} \int_{t}^{T} \int_{\mathbb{R}^{n}}\left\{2 V _ { x _ { k } } ^ { \varepsilon } \left[\frac{1}{2} \nabla \cdot\left(\sigma \sigma^{T}\left(V_{x_{k}}^{\varepsilon}\right)_{x}\right)+\left\langle\bar{b},\left(V_{x_{k}}^{\varepsilon}\right)_{x}\right\rangle+\bar{b}^{0} V_{x_{k}}^{\varepsilon}+\nabla \cdot\left(\sigma q_{x_{k}}^{\varepsilon}\right)\right.\right. \\
& +\left\langle\bar{\sigma}^{0}, q_{x_{k}}^{\varepsilon}\right\rangle-\psi^{\prime}\left(\frac{V^{\varepsilon}}{\varepsilon}\right) \frac{V_{x_{k}}^{\varepsilon}}{\varepsilon}+\frac{1}{2} \nabla \cdot\left(\left(\sigma \sigma^{T}\right)_{x_{k}} V_{x}^{\varepsilon}\right)+\left\langle\bar{b}_{x_{k}}, V_{x}^{\varepsilon}\right\rangle+\bar{b}_{x_{k}}^{0} V^{\varepsilon} \\
& \left.\left.+\nabla \cdot\left(\sigma_{x_{k}} q^{\varepsilon}\right)+\left\langle\bar{\sigma}_{x_{k}}^{0}, q^{\varepsilon}\right\rangle+\bar{g}_{x_{k}}\right]-\left|q_{x_{k}}^{\varepsilon}\right|^{2}\right\} d x d r \\
= & \mathbb{E} \int_{t}^{T} \int_{\mathbb{R}^{n}}\left\{\left|\sigma^{T}\left(V_{x_{k}}^{\varepsilon}\right)_{x}\right|^{2}+2\left\langle q_{x_{k}}^{\varepsilon}, \sigma^{T}\left(V_{x_{k}}^{\varepsilon}\right)_{x}-V_{x_{k}}^{\varepsilon} \bar{\sigma}^{0}\right\rangle\right. \\
& +\left|q_{x_{k}}^{\varepsilon}\right|^{2}+2\left\langle q^{\varepsilon}, \sigma_{x_{k}}^{T}\left(V_{x_{k}}^{\varepsilon}\right)_{x}-V_{x_{k}}^{\varepsilon} \bar{\sigma}_{x_{k}}^{0}\right\rangle+\left[(\nabla \cdot \bar{b})-2 \bar{b}^{0}\right]\left(V_{x_{k}}^{\varepsilon}\right)^{2}-2 V_{x_{k}}^{\varepsilon} \bar{g}_{x_{k}} \\
& +2 \psi^{\prime}\left(\frac{V^{\varepsilon}}{\varepsilon}\right) \frac{\left(V_{x_{k}}^{\varepsilon}\right)^{2}}{\varepsilon}+\left\langle\left(V_{x}^{\varepsilon}\right)_{x_{k}},\left(\sigma \sigma^{T}\right)_{x_{k}} V_{x}^{\varepsilon}\right\rangle \\
& \left.-2 V_{x_{k}}^{\varepsilon}\left\langle\bar{b}_{x_{k}}, V_{x}^{\varepsilon}\right\rangle+\bar{b}_{x_{k} x_{k}}^{0}\left(V^{\varepsilon}\right)^{2}\right\} d x d r .
\end{aligned}
$$

Copyright $@$ by SIAM. Unauthorized reproduction of this article is prohibited. 
Note that (recalling $\sigma=\left(\sigma_{1}, \ldots, \sigma_{d}\right)$, with each $\sigma_{i}$ taking values in $\mathbb{R}^{n}$ )

$$
\begin{aligned}
\nabla \cdot\left[V_{x_{k}}^{\varepsilon} \sigma_{x_{k}}\right]= & \left(\nabla \cdot\left[V_{x_{k}}^{\varepsilon}\left(\sigma_{1}\right)_{x_{k}}\right], \ldots, \nabla \cdot\left[V_{x_{k}}^{\varepsilon}\left(\sigma_{d}\right)_{x_{k}}\right]\right)^{T} \\
= & \left(\left\langle\left(V_{x_{k}}^{\varepsilon}\right)_{x},\left(\sigma_{1}\right)_{x_{k}}\right\rangle+V_{x_{k}}^{\varepsilon} \nabla \cdot\left[\left(\sigma_{1}\right)_{x_{k}}\right], \ldots,\right. \\
& \left.\left\langle\left(V_{x_{k}}^{\varepsilon}\right)_{x},\left(\sigma_{d}\right)_{x_{k}}\right\rangle+V_{x_{k}}^{\varepsilon} \nabla \cdot\left[\left(\sigma_{d}\right)_{x_{k}}\right]\right)^{T} \\
= & \sigma_{x_{k}}^{T}\left(V_{x_{k}}^{\varepsilon}\right)_{x}+V_{x_{k}}^{\varepsilon} \nabla \cdot\left(\sigma_{x_{k}}\right) .
\end{aligned}
$$

Hence (recall that $q_{x}^{\varepsilon}$ takes values in $\mathbb{R}^{d \times n}$ ),

$$
\begin{aligned}
\mathbb{E} \int_{t}^{T} \int_{\mathbb{R}^{n}}\left\langle q^{\varepsilon}, \sigma_{x_{k}}^{T}\left(V_{x_{k}}^{\varepsilon}\right)_{x}\right\rangle d x d r & =\mathbb{E} \int_{t}^{T} \int_{\mathbb{R}^{n}}\left\langle q^{\varepsilon}, \nabla \cdot\left[V_{x_{k}}^{\varepsilon} \sigma_{x_{k}}\right]-V_{x_{k}}^{\varepsilon} \nabla \cdot \sigma_{x_{k}}\right\rangle d x d r \\
& =-\mathbb{E} \int_{t}^{T} \int_{\mathbb{R}^{n}} V_{x_{k}}^{\varepsilon}\left\{\operatorname{tr}\left(\sigma_{x_{k}} q_{x}^{\varepsilon}\right)+\left\langle q^{\varepsilon}, \nabla \cdot \sigma_{x_{k}}\right\rangle\right\} d x d r .
\end{aligned}
$$

On the other hand,

$$
\begin{aligned}
{\left[\left\langle\left(\sigma \sigma^{T}\right)_{x_{k}} V_{x}^{\varepsilon}, V_{x}^{\varepsilon}\right\rangle\right]_{x_{k}}=} & \left\langle\left(\sigma \sigma^{T}\right)_{x_{k} x_{k}} V_{x}^{\varepsilon}, V_{x}^{\varepsilon}\right\rangle+\left\langle\left(\sigma \sigma^{T}\right)_{x_{k}}\left(V_{x}^{\varepsilon}\right)_{x_{k}}, V_{x}^{\varepsilon}\right\rangle \\
& +\left\langle\left(\sigma \sigma^{T}\right)_{x_{k}} V_{x}^{\varepsilon},\left(V_{x}^{\varepsilon}\right)_{x_{k}}\right\rangle \\
= & \left\langle\left(\sigma \sigma^{T}\right)_{x_{k} x_{k}} V_{x}^{\varepsilon}, V_{x}^{\varepsilon}\right\rangle+2\left\langle\left(\sigma \sigma^{T}\right)_{x_{k}} V_{x}^{\varepsilon},\left(V_{x}^{\varepsilon}\right)_{x_{k}}\right\rangle
\end{aligned}
$$

which implies that

$$
\mathbb{E} \int_{t}^{T} \int_{\mathbb{R}^{n}}\left\langle\left(V_{x}^{\varepsilon}\right)_{x_{k}},\left(\sigma \sigma^{T}\right)_{x_{k}} V_{x}^{\varepsilon}\right\rangle d x d r=-\frac{1}{2} \mathbb{E} \int_{t}^{T} \int_{\mathbb{R}^{n}}\left\langle\left(\sigma \sigma^{T}\right)_{x_{k} x_{k}} V_{x}^{\varepsilon}, V_{x}^{\varepsilon}\right\rangle d x d r .
$$

Thus, (7.33) can be written as

$$
\begin{aligned}
& -\mathbb{E} \int_{\mathbb{R}^{n}}\left|V_{x_{k}}^{\varepsilon}(t, x)\right|^{2} d x \\
= & \mathbb{E} \int_{t}^{T} \int_{\mathbb{R}^{n}}\left\{\left|\sigma^{T}\left(V_{x_{k}}^{\varepsilon}\right)_{x}\right|^{2}+2\left\langle q_{x_{k}}^{\varepsilon}, \sigma^{T}\left(V_{x_{k}}^{\varepsilon}\right)_{x}-V_{x_{k}}^{\varepsilon} \bar{\sigma}^{0}\right\rangle+\left|q_{x_{k}}^{\varepsilon}\right|^{2}-2 V_{x_{k}}^{\varepsilon} \operatorname{tr}\left(\sigma_{x_{k}} q_{x}^{\varepsilon}\right)\right. \\
& +2\left\langle q^{\varepsilon}, \bar{\sigma}_{x_{k}}^{0}-\nabla \cdot \sigma_{x_{k}}\right\rangle V_{x_{k}}^{\varepsilon}+\left[(\nabla \cdot \bar{b})-2 \bar{b}^{0}\right]\left(V_{x_{k}}^{\varepsilon}\right)^{2}-2 V_{x_{k}}^{\varepsilon} \bar{g}_{x_{k}}+2 \psi^{\prime}\left(\frac{V^{\varepsilon}}{\varepsilon}\right) \frac{\left(V_{x_{k}}^{\varepsilon}\right)^{2}}{\varepsilon} \\
& \left.-\frac{1}{2}\left\langle\left(\sigma \sigma^{T}\right)_{x_{k} x_{k}} V_{x}^{\varepsilon}, V_{x}^{\varepsilon}\right\rangle-2 V_{x_{k}}^{\varepsilon}\left\langle\bar{b}_{x_{k}}, V_{x}^{\varepsilon}\right\rangle+\bar{b}_{x_{k} x_{k}}^{0}\left(V^{\varepsilon}\right)^{2}\right\} d x d r .
\end{aligned}
$$

In what follows, we let

$$
\langle A, B\rangle=\operatorname{tr}\left(A B^{T}\right)=\sum_{k=1}^{n} a_{k}^{T} b_{k}, \quad \forall A \equiv\left(\begin{array}{c}
a_{1}^{T} \\
\vdots \\
a_{n}^{T}
\end{array}\right), B \equiv\left(\begin{array}{c}
b_{1}^{T} \\
\vdots \\
b_{n}^{T}
\end{array}\right) \in \mathbb{R}^{n \times m} .
$$

Then one has

$$
|A|^{2}=\operatorname{tr}\left(A^{T} A\right)=\sum_{i=1}^{n} \sum_{j=1}^{m}\left|a_{i j}\right|^{2} \quad \forall A \equiv\left(a_{i j}\right) \in \mathbb{R}^{n \times m} .
$$

Copyright $@$ by SIAM. Unauthorized reproduction of this article is prohibited. 
Now, summing (7.38) up with respect to $k$, we obtain (recall that $q_{x}$ takes values in $\mathbb{R}^{d \times n}$ )

$$
\begin{aligned}
& -\mathbb{E} \int_{\mathbb{R}^{n}}\left|V_{x}^{\varepsilon}(t, x)\right|^{2} d x \\
& =\sum_{k=1}^{n} \mathbb{E} \int_{t}^{T} \int_{\mathbb{R}^{n}}\left\{\left|\sigma^{T}\left(V_{x_{k}}^{\varepsilon}\right)_{x}\right|^{2}+2\left\langle q_{x_{k}}^{\varepsilon}, \sigma^{T}\left(V_{x_{k}}^{\varepsilon}\right)_{x}-V_{x_{k}}^{\varepsilon} \bar{\sigma}^{0}\right\rangle+\left|q_{x_{k}}^{\varepsilon}\right|^{2}-2 V_{x_{k}}^{\varepsilon} \operatorname{tr}\left(\sigma_{x_{k}} q_{x}^{\varepsilon}\right)\right. \\
& +2\left\langle q^{\varepsilon}, \bar{\sigma}_{x_{k}}^{0}-\nabla \cdot \sigma_{x_{k}}\right\rangle V_{x_{k}}^{\varepsilon}+\left[(\nabla \cdot \bar{b})-2 \bar{b}^{0}\right]\left(V_{x_{k}}^{\varepsilon}\right)^{2}-2 V_{x_{k}}^{\varepsilon} \bar{g}_{x_{k}}+2 \psi^{\prime}\left(\frac{V^{\varepsilon}}{\varepsilon}\right) \frac{\left(V_{x_{k}}^{\varepsilon}\right)^{2}}{\varepsilon} \\
& \left.-\frac{1}{2}\left\langle\left(\sigma \sigma^{T}\right)_{x_{k} x_{k}} V_{x}^{\varepsilon}, V_{x}^{\varepsilon}\right\rangle-2 V_{x_{k}}^{\varepsilon}\left\langle\bar{b}_{x_{k}}, V_{x}^{\varepsilon}\right\rangle+\bar{b}_{x_{k} x_{k}}^{0}\left(V^{\varepsilon}\right)^{2}\right\} d x d r \\
& =\mathbb{E} \int_{t}^{T} \int_{\mathbb{R}^{n}}\left\{\left|\sigma^{T} V_{x x}^{\varepsilon}\right|^{2}+2 \operatorname{tr}\left[\left(q_{x}^{\varepsilon}\right)^{T} \sigma^{T} V_{x x}^{\varepsilon}\right]+\left|q_{x}^{\varepsilon}\right|^{2}\right. \\
& -2 \operatorname{tr}\left[\left(q_{x}^{\varepsilon}\right)^{T}\left(\bar{\sigma}^{0} V_{x}^{\varepsilon}-\sum_{k=1}^{n} V_{x_{k}}^{\varepsilon} \sigma_{x_{k}}^{T}\right)\right]+2\left\langle q^{\varepsilon},\left(\bar{\sigma}^{0}-\nabla \cdot \sigma\right)_{x} V_{x}^{\varepsilon}\right\rangle \\
& +\left[(\nabla \cdot \bar{b})-2 \bar{b}^{0}\right]\left|V_{x}^{\varepsilon}\right|^{2}-2\left\langle V_{x}^{\varepsilon}, \bar{g}_{x}\right\rangle+2 \psi^{\prime}\left(\frac{V^{\varepsilon}}{\varepsilon}\right) \frac{\left|V_{x}^{\varepsilon}\right|^{2}}{\varepsilon} \\
& \left.-\frac{1}{2}\left\langle\left[\Delta\left(\sigma \sigma^{T}\right)\right] V_{x}^{\varepsilon}, V_{x}^{\varepsilon}\right\rangle-2\left\langle\bar{b}_{x} V_{x}^{\varepsilon}, V_{x}^{\varepsilon}\right\rangle+\left(\Delta \bar{b}^{0}\right)\left|V^{\varepsilon}\right|^{2}\right\} d x d r \\
& =\mathbb{E} \int_{t}^{T} \int_{\mathbb{R}^{n}}\left\{\left|\sigma^{T} V_{x x}^{\varepsilon}+q_{x}^{\varepsilon}-\bar{\sigma}^{0} V_{x}^{\varepsilon}-\sum_{k=1}^{n} V_{x_{k}}^{\varepsilon} \sigma_{x_{k}}^{T}\right|^{2}-\left|\bar{\sigma}^{0} V_{x}^{\varepsilon}+\sum_{k=1}^{n} V_{x_{k}}^{\varepsilon} \sigma_{x_{k}}^{T}\right|^{2}\right. \\
& +2 \operatorname{tr}\left[V_{x x}^{\varepsilon}\left(\sigma \bar{\sigma}^{0} V_{x}^{\varepsilon}+\sum_{k=1}^{n} V_{x_{k}}^{\varepsilon} \sigma \sigma_{x_{k}}^{T}\right)\right]+2\left\langle q^{\varepsilon},\left(\bar{\sigma}^{0}-\nabla \cdot \sigma\right)_{x} V_{x}^{\varepsilon}\right\rangle-2\left\langle V_{x}^{\varepsilon}, \bar{g}_{x}\right\rangle \\
& \left.+2 \psi^{\prime}\left(\frac{V^{\varepsilon}}{\varepsilon}\right) \frac{\left|V_{x}^{\varepsilon}\right|^{2}}{\varepsilon}-\left\langle\left[\frac{1}{2} \Delta\left(\sigma \sigma^{T}\right)\right]+\bar{b}_{x}+\bar{b}_{x}^{T}-\left((\nabla \cdot \bar{b})-2 \bar{b}^{0}\right) I\right] V_{x}^{\varepsilon}, V_{x}^{\varepsilon}\right\rangle \\
& \left.+\left(\Delta \bar{b}^{0}\right)\left|V^{\varepsilon}\right|^{2}\right\} d x d r \text {. }
\end{aligned}
$$

Note that

$$
\begin{aligned}
\mathbb{E} \int_{t}^{T} \int_{\mathbb{R}^{n}} \operatorname{tr}\left[V_{x x}^{\varepsilon} \sigma \bar{\sigma}^{0}\left(V_{x}^{\varepsilon}\right)^{T}\right] d x d r & =\mathbb{E} \int_{t}^{T} \int_{\mathbb{R}^{n}}\left(V_{x x}^{\varepsilon} V_{x}^{\varepsilon}\right)^{T} \sigma \bar{\sigma}^{0} d x d r \\
& =\frac{1}{2} \mathbb{E} \int_{t}^{T} \int_{\mathbb{R}^{n}}\left[\left(\left|V_{x}^{\varepsilon}\right|^{2}\right)_{x}\right]^{T} \sigma \bar{\sigma}^{0} d x d r \\
& =-\frac{1}{2} \mathbb{E} \int_{t}^{T} \int_{\mathbb{R}^{n}}\left|V_{x}^{\varepsilon}\right|^{2}\left(\nabla \cdot\left[\sigma \bar{\sigma}^{0}\right]\right) d x d r \\
& \geq-C \mathbb{E} \int_{t}^{T} \int_{\mathbb{R}^{n}}\left|V_{x}^{\varepsilon}\right|^{2} d x d r
\end{aligned}
$$

Also, we note that

$$
\operatorname{tr}\left[V_{x x}^{\varepsilon} \sigma \sigma_{x_{k}}^{T}\right]=\operatorname{tr}\left[\left(V_{x x}^{\varepsilon} \sigma \sigma_{x_{k}}^{T}\right)^{T}\right]=\operatorname{tr}\left[\sigma_{x_{k}} \sigma^{T} V_{x x}^{\varepsilon}\right]=\operatorname{tr}\left[V_{x x}^{\varepsilon} \sigma_{x_{k}} \sigma^{T}\right]
$$

Copyright (C) by SIAM. Unauthorized reproduction of this article is prohibited. 
Now, if we denote $\Phi_{k}=\sigma \sigma_{x_{k}}^{T}+\sigma_{x_{k}} \sigma^{T}$, then it is $\mathbb{R}^{n \times n}$-valued, symmetric, and

$$
\begin{aligned}
\operatorname{tr}\left[V_{x x}^{\varepsilon} V_{x_{k}}^{\varepsilon} \Phi_{k}\right]= & \operatorname{tr}\left[\left(V_{x_{k}}^{\varepsilon} \Phi_{k} V_{x}^{\varepsilon}\right)_{x}\right]-\left\langle\nabla \cdot\left(V_{x_{k}}^{\varepsilon} \Phi_{k}\right), V_{x}^{\varepsilon}\right\rangle \\
= & \nabla \cdot\left(V_{x_{k}}^{\varepsilon} \Phi_{k} V_{x}^{\varepsilon}\right)-\left\langle V_{x_{k}}^{\varepsilon} \nabla \cdot \Phi_{k}, V_{x}^{\varepsilon}\right\rangle-\left\langle\Phi_{k}\left(V_{x}^{\varepsilon}\right)_{x_{k}}, V_{x}^{\varepsilon}\right\rangle \\
= & \nabla \cdot\left(V_{x_{k}}^{\varepsilon} \Phi_{k} V_{x}^{\varepsilon}\right)-\left\langle V_{x_{k}}^{\varepsilon} \nabla \cdot \Phi_{k}, V_{x}^{\varepsilon}\right\rangle-\frac{1}{2}\left[\left\langle\Phi_{k} V_{x}^{\varepsilon}, V_{x}^{\varepsilon}\right\rangle\right]_{x_{k}} \\
& +\frac{1}{2}\left\langle\left(\Phi_{k}\right)_{x_{k}} V_{x}^{\varepsilon}, V_{x}^{\varepsilon}\right\rangle .
\end{aligned}
$$

Thus,

$$
\begin{aligned}
\mathbb{E} \int_{t}^{T} \int_{\mathbb{R}^{n}} \operatorname{tr}\left[V_{x x}^{\varepsilon} \sum_{k=1}^{n} V_{x_{k}}^{\varepsilon} \sigma \sigma_{x_{k}}^{T}\right] d x d r \\
=\frac{1}{2} \mathbb{E} \int_{t}^{T} \int_{\mathbb{R}^{n}} \operatorname{tr}\left[V_{x x}^{\varepsilon} \sum_{k=1}^{n} V_{x_{k}}^{\varepsilon}\left(\sigma \sigma_{x_{k}}^{T}+\sigma_{x_{k}} \sigma^{T}\right)\right] d x d r \\
=\mathbb{E} \int_{t}^{T} \int_{\mathbb{R}^{n}}\left\{-\frac{1}{2}\left\langle\sum_{k=1}^{n} V_{x_{k}}^{\varepsilon} \nabla \cdot\left[\sigma \sigma_{x_{k}}^{T}+\sigma_{x_{k}} \sigma^{T}\right], V_{x}^{\varepsilon}\right\rangle\right. \\
\left.\quad+\frac{1}{4}\left\langle\left(\sum_{k=1}^{n}\left[\sigma \sigma_{x_{k}}^{T}+\sigma_{x_{k}} \sigma^{T}\right]_{x_{k}}\right) V_{x}^{\varepsilon}, V_{x}^{\varepsilon}\right\rangle\right\} d x d r \\
\geq-C \mathbb{E} \int_{t}^{T} \int_{\mathbb{R}^{n}}\left|V_{x}^{\varepsilon}\right|^{2} d x d r .
\end{aligned}
$$

Consequently, making use of (5.18) with $m=1$, we obtain

$$
\begin{aligned}
-\mathbb{E} \int_{\mathbb{R}^{n}}\left|V_{x}^{\varepsilon}(t, x)\right|^{2} d x \geq & \mathbb{E} \int_{t}^{T} \int_{\mathbb{R}^{n}}\left\{\left|\sigma^{T} V_{x x}^{\varepsilon}+q_{x}^{\varepsilon}-\bar{\sigma}^{0}\left(V_{x}^{\varepsilon}\right)^{T}-\sum_{k=1}^{n} V_{x_{k}}^{\varepsilon} \sigma_{x_{k}}^{T}\right|^{2}-\left|q^{\varepsilon}\right|^{2}\right. \\
& \left.+2 \psi^{\prime}\left(\frac{V^{\varepsilon}}{\varepsilon}\right) \frac{\left|V_{x}^{\varepsilon}\right|^{2}}{\varepsilon}-C\left|V_{x}^{\varepsilon}\right|^{2}-C\left|V^{\varepsilon}\right|^{2}-\left|\bar{g}_{x}\right|^{2}\right\} d x d r \\
\geq & \mathbb{E} \int_{t}^{T} \int_{\mathbb{R}^{n}}\left\{\left|\sigma^{T} V_{x x}^{\varepsilon}+q_{x}^{\varepsilon}\right|^{2}+2 \psi^{\prime}\left(\frac{V^{\varepsilon}}{\varepsilon}\right) \frac{\left|V_{x}^{\varepsilon}\right|^{2}}{\varepsilon}\right. \\
& \left.-C\left|V_{x}^{\varepsilon}\right|^{2}-\left|\bar{g}_{x}\right|^{2}-C|\bar{g}|^{2}\right\} d x d r .
\end{aligned}
$$

By Gronwall's inequality, we obtain (5.22).

Acknowledgment. The authors would like to thank the anonymous referee for his/her suggestive comments, which lead to the current much better version of the paper.

\section{REFERENCES}

[1] M. Beibel And H. R. Lerche, Optimal stopping of regular diffusion under random discounting, Theory Probab. Appl., 45 (2001), pp. 547-557.

[2] A. Bensoussan, Stochastic Control by Functional Analysis Methods, North-Holland, Amsterdam, 1982.

[3] J. M. Bismut and B. Skalli, Temps d'arrêt, thérie générale de processus et processus de Markov, Z. Wahrsch. verw. Gebiete, 39 (1977), pp. 301-313.

[4] R. Buckdahn And J. Ma, Pathwise stochastic control problems and stochastic HJB equations, SIAM J. Control Optim., 45 (2007), pp. 2224-2256.

Copyright $@$ by SIAM. Unauthorized reproduction of this article is prohibited. 
[5] S. Dayanik and I. Karatzas, On the optimal stopping problem for one-dimensional diffusions, Stochastic Process. Appl., 107 (2003), pp. 173-212.

[6] C. Dellarcherie, Capacités et Processus Stochastiques, Springer-Verlag, New York, 1972.

[7] N. Egglezos and I. Karatzas, Aspects of utility maximization with habit formation: Dynamic programming and stochastic PDE's, preprint, Columbia University, New York, 2007.

[8] N. El KARoui, Les aspects probabilistes du contrôle stochastique, in Ecole d'Été de Probabilités de Saint-Flour IX-1979, Lecture Notes in Math. 876, Springer-Verlag, New York, pp. 73-238.

[9] A. G. FAKEEV, Optimal stopping rules for processes with continuous parameter, Theory Probab. Appl., 15 (1970), pp. 324-331.

[10] A. G. FAKEev, Optimal stopping of a Markov process, Theory Probab. Appl., 16 (1971), pp. 694696.

[11] R. Fernholz And I. Karatzas, Stochastic portfolio theory: An overview, preprint, Columbia University, New York, 2008.

[12] A. Friedman, Variational Inequalities and Free Boundary Problems, John Wiley \& Sons, New York, 1983.

[13] I. Karatzas and S. Shreve, Brownian Motion and Stochastic Calculus, Springer-Verlag, New York, 1998.

[14] I. Karatzas And S. Shreve, Methods of Mathematical Finance, Springer-Verlag, New York, 1998.

[15] H. Kunita, Stochastic Flows and Stochastic Differential Equations, Cambridge University Press, London, 1990.

[16] J. Ma AND J. Yong, On linear, degenerate backward stochastic partial differential equations, Probab. Theory Related Fields, 113 (1999), pp. 135-170.

[17] J. Ma And J. Yong, Forward-Backward Stochastic Differential Equations and Their Applications, Springer-Verlag, Berlin, 1999.

[18] J. Ma AND J. Yong, Dynamic programming for multidimensional stochastic control problems, Acta Math. Sinica, 15 (1999), pp. 485-506.

[19] A. Nikeghbali, An essay on the general theory of stochastic processes, Prob. Surv., 3 (2006), pp. 345-412.

[20] E. Pardoux And A. Rascanu, Backward stochastic variational inequalities, Stoch. Stoch. Rep., 67 (1999), pp. 159-167.

[21] S. Peng, Stochastic Hamilton-Jacobi-Bellman equations, SIAM J. Control Optim., 30 (1992), pp. 284-304.

[22] S. PENG, Backward stochastic differential equations and applications to optimal control, Appl. Math. Optim., 27 (1993), pp. 125-144.

[23] G. Peskir And A. Shiryaev, Optimal Stopping and Free-Boundary Problems, BirkhäuserVerlag, Basel, 2006.

[24] A. N. Shirayaev, Optimal Stopping Rules, Springer-Verlag, New York, 1978.

[25] M. E. Thompson, Continuous parameter optimal stopping problems, Z. Wahrsch. verw. Gebiete, 19 (1971), pp. 302-318.

[26] S. TANG, Semi-linear systems of backward stochastic partial differential equations in $\mathbb{R}^{n}$, Chinese Ann. Math. Ser. B, 26 (2005), pp. 437-456.

[27] S. TANG AND S. H. Hou, Switching games of stochastic differential systems, SIAM J. Control Optim., 46 (2007), pp. 900-929.

[28] S. TANG AND J. YONG, Finite horizon stochastic optimal switching and impulse controls with a viscosity solution approach, Stoch. Stoch. Rep., 45 (1993), pp. 145-176.

[29] J. Yong And X. Y. Zhou, Stochastic Controls: Hamiltonian Systems and HJB Equations, Springer-Verlag, New York, 1999.

Copyright $@$ by SIAM. Unauthorized reproduction of this article is prohibited. 